\title{
VARIATIONAL AND TOPOLOGICAL METHODS IN NONLINEAR PROBLEMS ${ }^{1}$
}

\author{
BY L. NIRENBERG
}

These lectures are meant as an informal introduction to some of the techniques used in proving existence of solutions of nonlinear problems of the form

$$
F(u)=y .
$$

Here $F$ is a continuous (and usually smooth) mapping from one topological space $X$ to another $Y$, and the spaces are usually infinite dimensional. The model to keep in mind is one in which these spaces are function spaces defined in domains on finite-dimensional manifolds, and $F$ is a system of nonlinear partial differential operators-or integral operators.

A number of special topics will be presented-in three parts:

I. Global methods: homotopy, in particular topological degree theory, and extensions. Applications to nonlinear boundary value problems.

II. Variational methods, in which a solution is a stationary point of some functional. Applications.

III. Local study, perturbation about a solution.

An important analytic aspect of all these problems is that of finding a priori estimates for the solutions. How one does that varies from problem to problem and I will barely touch on these technical aspects. I will try, rather, to avoid technicalities and stress the topological and variational ideas.

The lectures are not addressed to the experts in these fields-for them there will be little new. They are given with the hope of attracting others to the subject. Up to now, the topological and abstract ideas used are rather primitive, and I am confident that there will be enormous further development-involving more and more sophisticated topology.

A condensed version of some of this material was presented in [48].

Here is a more specific list of the topics treated.

I.1 Some classical things. Continuity method. Degree theory.

I. 2 Some recent extensions of Leray-Schauder degree theory.

I.3 Extension of degree theory to Fredholm maps by Elworthy and Tromba.

Received by the editors October 20, 1980.

1980 Mathematics Subject Classification. Primary 35-02, 35-A15, 35A05, 35B10, 4902, 4602, 58F05, 58-G16; Secondary 35B32, 35J65, 35L20, 58E15.

${ }^{1}$ Based on four lectures given in March 1980 at the Institute for Advanced Study in the Hermann Weyl lecture series. The work was partially supported by National Science Foundation Grants MCS-7900813 and INT-77-20878 and by U. S. Army Research Office Grant DAA-29-78G-0127. 
I.4 Fredholm maps with positive index.

I.5 Modifications of degree associated to invariant orbits of ordinary differential equations.

II.1 The Palais-Smale condition (PS) and an elliptic boundary Value problem.

II. 2 The mountain pass lemma. Solution of the boundary value problem.

II.3 Generalizations and variants of the mountain pass lemma.

II.4 A theorem of P. Rabinowitz on periodic solutions for a Hamiltonian system.

II.5 Periodic solutions of a nonlinear string equation.

III.1 Remarks on bifurcation theory for Fredholm operators.

III.2 The Nash-Moser Implicit Function technique.

III.3 Klainerman's result on the existence of smooth solutions for all time of a nonlinear hyperbolic initial value problem.

\section{Global topological methods}

For convenience we will assume that the spaces $X, Y$ are Banach spaces and that $F$ is a smooth mapping. We begin with some very classical things.

I.1 A standard, but still very useful, method, is to try to show that

(i) Range of $F$ is open,

(ii) Range of $F$ is closed.

For (i), a natural tool is the implicit function theorem. If $y=F\left(u_{0}\right)$, in order to show that a neighbourhood of $y_{0}$ is contained in the image of one of $u_{0}$, one looks at the continuous linear operator $A=F^{\prime}\left(u_{0}\right): X \rightarrow Y$. If $A$ is bijective the desired result follows from the implicit function theorem (IFT). It suffices, in fact, that $A$ be surjective.

We shall make use of the following

Definition. If $A=F^{\prime}\left(u_{0}\right)$ is bijective, the point $u_{0}$ is called a regular point of $F$.

To establish (ii) one usually tries to show that the map $F$ is proper, i.e., the preimage of every compact set is compact. It is here that a priori estimates for solutions of the equation enter in a crucial way. This is usually where the hard work comes in.

A variant of this approach is the

ConTINUITY METHOD. The operator $F$ is continuously connected by a one-parameter family of operators $F_{t}, 0 \leqslant t \leqslant 1, F_{1}=F$, to an operator $F_{0}$ for which it is known that a solution of (1) exists. Then one tries to prove

(i') the set of $t$ for which a solution exists is open.

(ii') the set of $t$ for which a solution exists is closed.

Again one leans on the implicit function theory and on a priori estimates.

A word of caution: For a nonlinear partial differential operator $F$ these methods usually fail-unless the operator is elliptic (to be explained later for a special case). The reason is that the implicit function theorem may not be applicable. There are of course different ways in which it may fail: (a) The linear operator $A=F^{\prime}\left(u_{0}\right)$ may be injective, and so invertible on its range, 
but the range may not be the whole space $Y$. For example the range may be dense but not closed in $Y$. This may happen in the following way. It may be that one can solve (in some sense) $A u=y$ for every $y \in Y$, but the "solution" $x$ doesn't lie in the space $X$, but in some larger space. For example, $X$ and $Y$ may be spaces of functions with some given degrees of smoothness-defined on some bounded domain in $R^{n}$. It may be that we can solve $A u=y$, $\forall y \in Y$, but $u$ does not have the desired smoothness, i.e. we "lose derivatives". This is typical for nonelliptic differential operators. In such cases one tries to use the generalized implicit function theorems, such as the Nash-Moser method. This will be taken up in part III.

(b) The IFT may fail in a simpler way. One may have

$$
\operatorname{ker} F^{\prime}\left(u_{0}\right) \neq 0
$$

but

$$
\text { Range } F^{\prime}\left(u_{0}\right) \text { is a closed subspace of } Y \text {. }
$$

In such a situation the local problem is usually called a bifurcation problem (splitting of solutions may occur). The cases that have received the most intensive study are those in which

$$
\operatorname{dim} \operatorname{ker} F^{\prime}\left(u_{0}\right)=d<\infty
$$

and

$$
\text { codim Range } F^{\prime}\left(u_{0}\right)=d^{\prime}<\infty
$$

i.e. $Y$ may be decomposed as Range $F^{\prime}\left(u_{0}\right) \oplus Y_{2}$, $\operatorname{dim} Y_{2}=d^{\prime}$. If $F$ has this property at $u_{0}$ we say that $F$ is a Fredholm operator there, and its index is $d-d^{\prime}$.

Using well-known properties of linear Fredholm operators (see for example [56]) one finds that $F$ is Fredholm everywhere in a neighbourhood of $u_{0}$ and its index is constant there.

Turning to topological methods, recall that homotopy theory began with attempts to find conditions on maps $F$ to guarantee that (1) has a solution. Consider a continuous map $F$ from the closed unit ball $B$ in $R^{n}$ into $R^{k}$ for which one wishes to solve the equation

$$
F(u)=0 .
$$

Let $\phi$ be the restriction of $F$ to $\partial B$ and assume $\phi: \partial B \rightarrow R^{k} \backslash\{0\}$. Homotopy theory yields a sufficient condition on $\phi$ so that every continuous extension $F$ of $\phi$ to the inside of $B$ has, necessarily, a solution of $F(u)=0$. Namely, the homotopy class of $\phi: S^{n-1} \rightarrow R^{k} \backslash\{0\}$ should be nontrivial. An equivalent formulation, for the normalized map

$$
\psi(u)=\frac{\phi(u)}{|\phi(u)|}
$$

is that $\psi: S^{n-1} \rightarrow S^{k-1}$ should be homotopically nontrivial. In case $k=n$ this is equivalent to the assertion that the degree of the map $\psi=$ the degree of the map $F$ at the origin, is nonzero. For generic $y$ with $F: \partial B \rightarrow R^{n} \backslash\{y\}$, the 
degree of the map $F$ at $y$,

$$
\operatorname{deg}(F, B, y)=\nu, \quad \text { an integer. }
$$

$\nu$ represents the number of times $y$ is covered, i.e. the number of preimages of $y$ with each preimage counted with + or -1 according as, locally, $F$ preserves or reverses orientation there.

The most frequently used topological tool in attacking global nonlinear problems is the Leray, Schauder [38] extension of degree theory to infinite dimensions. In Banach space $X$ it applies to operators $F: X \rightarrow X$ of a special form

$$
F=I-K
$$

where $I=$ Identity operator and $K$ is a compact operator (i.e. the closure of the image under $K$ of any bounded set is compact). If $K$ is smooth then $F$ is a Fredholm map everywhere with index $=0$.

Let us consider equation (1) for such $F$ defined in the closure of a bounded domain $\Omega$ in $X$, mapping into $X$. Assume

$$
y \notin F(\partial \Omega) \text {. }
$$

Then the degree of the map $F$ in $\bar{\Omega}$ at the point $y$ is defined:

$$
\operatorname{deg}(F, \Omega, y)=\nu=\text { integer. }
$$

It is obtained from finite-dimensional degree by approximating $K$ (within $\varepsilon$ on $\bar{\Omega}$ ) by a map $K_{\varepsilon}$ into a finite-dimensional subspace $X_{\varepsilon}$ containing $y$. One then defines $\operatorname{deg}(F, \Omega, y)$ as the finite-dimensional degree

$$
\operatorname{deg}\left(\left(I-K_{\varepsilon}\right), \Omega \cap X_{\varepsilon}, y\right) .
$$

To verify that this is independent of $\varepsilon$, for small $\varepsilon$, one uses the fact that degree does not change under suspension of a map $F$, i.e. by extension of the map to a product space with another space by taking the product of $F$ with the identity map in the other space.

We list a few important properties:

(a) If $\nu=\operatorname{deg}(F, \Omega, y) \neq 0$ then $F(u)=y$ has a solution in $\Omega$. For $F=I$, $\nu=1$.

(b) If $\bar{\Omega}=\cup_{i=1}^{\infty} \bar{\Omega}_{i}, \Omega_{i}$ open, nonoverlapping and $y \notin F\left(\partial \Omega_{i}\right), \forall i$, then

$$
\operatorname{deg}(F, \Omega, y)=\sum_{i} \operatorname{deg}\left(F, \Omega_{i}, y\right)
$$

(c) The degree $\nu$ is invariant under homotopy of the map $F_{t}=I-K_{t}$, $0 \leqslant t \leqslant 1$, in our class, i.e., provided $\left\{K_{t}(x) \mid x \in \bar{\Omega}, 0 \leqslant t \leqslant 1\right\}$ has compact closure and

$$
y \notin F_{t}(\partial \Omega), \quad \forall t .
$$

(d) Suppose that a solution $u$ of $F(u)=u-K(u)=y$ is a regular point of $F$. (By IFT it is an isolated solution.) Then the local degree (index) of $F$ at $u$ is defined as $\operatorname{ind}(u)=\operatorname{loc} \operatorname{deg} F$ at $u=\operatorname{deg}(F, \varepsilon$-ball about $u ; y)$. It is independent of $\varepsilon$ for $\varepsilon$ small, and equals +1 or -1 according as the sum of the algebraic multiplicities of the negative eigenvalues of $F^{\prime}(u)$ is even or odd.

Presentations of degree theory and derivations on these properties may be found in [37], [57], [39], [47], [51] and many other places. 
Degree theory is often used in the following way: To show that (1) has a solution with $F$ as in (2), try to show that all solutions lie inside some bounded domain $\Omega$-that is, obtain a priori estimates for all solutions. Then, to prove $\nu=\operatorname{deg}(F, \Omega, y) \neq 0$, construct a deformation of $F, F_{t}=I-K_{t}$ belonging to our class, and such that $y \notin F_{t}(\partial \Omega)$, deforming $F=F_{1}$ to $F_{0}$, for which one knows $\operatorname{deg}\left(F_{0}, \Omega, y\right) \neq 0$. For $F_{0}=I$, we find $\nu=1$.

Property (d) is sometimes useful in trying to determine the number of solutions of (1). Sometimes one can show that $F^{-1}(y)$ consists of a finite number of regular points in $\Omega$, all having the same local degrees, say \pm 1 . If $\operatorname{deg}(F, \Omega, y)=k$ it follows that there are exactly $|k|$ solutions in $\Omega$.

This completes the classical things and we now take up some recent extensions.

I.2 Considering, still, mappings of the form (2) and satisfying (3) we will describe two results related to $(\mathrm{d})$.

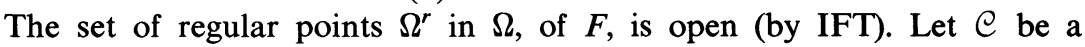
connected component of $\Omega^{r}$. For any $u \in \mathcal{C}$, define for small $\varepsilon$

$$
\operatorname{ind}(u)=(\operatorname{deg} F, \varepsilon \text {-ball about } u, F(u))= \pm 1 \text {. }
$$

It is easy to see that this is a constant in $\mathcal{C}$ and so may be considered as ind(e). In [7] Ambrosetti and Mancini showed (in a Hilbert space, though it works as well in a Banach space), that this index necessarily changes as we cross from one component of $\Omega^{r}$ to another-in a particular generic circumstance. They then applied this to prove that certain equations in Hilbert space have exactly three solutions. Their generic condition is easy to describe:

Let $u_{0} \in \Omega$ be a nonregular point of $F$ and assume that

(i) ker $F^{\prime}\left(u_{0}\right)$ is spanned by a vector $v$. Consequently Range $F^{\prime}\left(u_{0}\right)$ is a closed linear subspace $X_{1}$ of $X$, of codimension one. Consider next the second derivative (Hessian) $F^{\prime \prime}\left(u_{0}\right)$ of $F$. This is a symmetric bilinear map of $X \times X \rightarrow X$. Assume

(ii) there is a vector $w$ such that

$$
F^{\prime \prime}\left(u_{0}\right)(v, w) \text { is not in } X_{1} \text {. }
$$

In this case it is easy to see (Theorem 3.7.2 in [47]) that in a neighbourhood of $u_{0}$, the set $\Omega^{r}$ has two components which are separated by a smooth hypersurface. Their result is that under conditions (i), (ii), the indices of these two components are different.

We turn now to a recent generalization of (d). In bifurcation theory one sometimes encounters a finite-dimensional manifold of solutions of (1). Let us suppose that a connected component of $F^{-1}(y)$ consists of a compact finite-dimensional manifold $M$ without boundary. It is natural to try to determine $\operatorname{ind}(M)=$ the local degree at $y$ of $F$ in a neighbourhood of $M$-assuming $F$ is "regular" on $M$, i.e., assuming:

REGULARITY.

$$
\operatorname{ker} F^{\prime}(u)=T_{u} M, \quad \forall u \in M .
$$

Here $T_{u} M$ is the tangent space to $M$ at $u$. 
Assuming $M$ is orientable, and $F$ is regular on $M$, Sylvester [58] proved the following

THeOREM. If $\operatorname{dim} M$ is odd then ind $M=0$. If $\operatorname{dim} M$ is even then

$$
\begin{aligned}
\operatorname{ind}(M) & = \pm \chi(E) \\
& = \pm \text { Euler characteristic of a vector bundle } E \text { over } M,
\end{aligned}
$$

where for $u \in M$, the fibre

$$
E_{u}=X /\left(\text { Range } F^{\prime}(u)\right) .
$$

In particular, if $T_{u} M \cap$ Range $F^{\prime}(u)=0, \forall u \in M$, then

$$
\operatorname{ind}(M)= \pm \chi(M) \text {. }
$$

Furthermore one has + or - according as the sum of algebraic multiplicities of negative eigenvalues of $F^{\prime}(u)$ is even or odd, for $u \in M$.

So far no application has been made of the result.

Degree theory extends to set-valued maps of the form (2): For $u \in \bar{\Omega}, K(u)$ is assumed to be a compact convex set which is upper semicontinuous in $u$. Furthermore $\cup_{u \in \bar{\Omega}} K(u)$ has compact closure. One wishes to solve

$$
y \in u-K(u) \text {. }
$$

If $\forall u$ on $\partial \Omega, u-y \notin K(u)$, then $\operatorname{deg}(F, \Omega, y)$ can be defined as before, with similar properties. A treatment of this may be found in the book of Lloyd [39].

Recently this has been used effectively by K. C. Chang [18] to solve several problems: (a) plasma problems, (b) an obstacle problem, in which one wishes to find solutions of an elliptic partial differential equation satisfying a side condition: it is to lie above a given obstacle.

I.3 Attempts have been made to extend degree theory to mappings $F$ which do not have the form (2). Elworthy and Tromba [27], [28] have developed degree theory for smooth Fredholm mappings between Banach manifolds. (A Banach manifold modeled on a Banach space $X$ is an infinite-dimensional manifold for which each point has a distinguished neighbourhood $U$ and a chart map $\phi: U$ onto $X$.) For an overlapping neighbourhood $V$, with chart map $\psi: V \rightarrow X$, it is required that on $\psi(U \cap V), \phi \circ \psi^{-1}$ have the form (2). For convenience, we will consider only Fredholm maps between Banach spaces, $F: X \rightarrow Y$ with $F$ defined on the closure $\bar{\Omega}$ of a bounded domain $\Omega$ in $X . F$ is assumed to be proper.

Using suitable orientations on $X$ and $Y$, in terms of the admissible chart mappings, they defined for Fredholm maps $F$ of index zero, an oriented degree

$$
\operatorname{deg}(F, \Omega, y)=\nu, \quad \nu \text { an integer, }
$$

in case $y \notin F(\partial \Omega)$.

They proved that this has the properties (a)-(d) above except that (c) holds in a weaker form. Namely, under a suitably restricted class of deformations, the degree $\nu$ is not necessarily invariant, but $|\nu|$ is.

Their theory makes use of the Sard-Smale lemma. This has been redone in 
a somewhat simpler way, via more systematic use of transversality, by Borisovich, Zvyagin and Sapronov. See their useful expository article [12]. Furthermore they extend the Elworthy-Tromba theory to maps of the form $F=F_{1}+K$ where $F_{1}$ is a smooth Fredholm map of index zero and $K$ is a continuous compact map.

This generalized degree has not received much application but I believe it will prove useful. As an indication of its use in a situation where the Leray-Schauder degree does not apply let us consider a second order quasilinear elliptic equation for a real function $u(x)$ defined in a bounded domain $G$ in $R^{n}$ with smooth boundary-under the boundary condition $u=0$ on $\partial G$.

$$
\sum a^{i j}(x, u, \operatorname{grad} u) u_{x_{i} x_{j}}+f(x, u, \operatorname{grad} u)=0 \text { on } G .
$$

Ellipticity means that the quadratic form

$$
a^{i j}(x, u, \operatorname{grad} u) \xi_{i} \xi_{j}
$$

is always positive definite. We try to find a solution $u$ as a fixed point of the map $K: u \mapsto v$, where $v$ is the solution of

$$
\sum a^{i j}(x, u, \operatorname{grad} u) v_{x_{i} x_{j}}+f(x, u, \operatorname{grad} u)=0 \text { in } G, \quad v=0 \text { on } \partial G .
$$

Thus we assume we know how to solve linear elliptic boundary value problems. In appropriate function spaces, the map $K$ is a smoothing operator and therefore compact. For example if $u$ belongs to the Sobolev space $\boldsymbol{H}^{\boldsymbol{s}}$ of functions having square integrable derivatives in $G$ up to order $s$, for $s$ large then $v$ belongs to $H^{s+1}$.

So we wish to solve

$$
F(u)=u-K(u)=0 .
$$

Suppose we can establish a priori estimates for solutions together with derivatives-guaranteeing that the solutions all lie inside some big ball $B$ in our function space. Then $\operatorname{deg}(F, B, 0)$ is defined, and we may try to prove it is not zero.

In place of the quasilinear equation above let us consider a general nonlinear elliptic equation

$$
A[u] \equiv A\left(x, u, \operatorname{grad} u, u_{x_{i} x_{j}}\right)=y(x) \text { in } G, \quad u=0 \text { on } \partial G .
$$

Ellipticity means the quadratic forms

$$
\sum \frac{\partial A}{\partial u_{x_{i} x_{j}}}(x, \ldots) \xi_{i} \xi_{j}
$$

are all positive definite. It appears that the Leray-Schauder theory is not applicable since it does not seem possible to recast this in the form (1), (2). However in the space of functions in $H^{s}(G), s$ large, vanishing on the boundary, the Elworthy-Tromba theory of Fredholm maps may be applicable provided we have a priori bounds in $H^{s}$ for all solutions. Their degree

$$
\operatorname{deg}(A, B, y)=\nu
$$

is then defined, and $\nu \neq 0$ ensures the existence of a solution. It seems surprising that up to now this method has not been employed. 
I.4 Elworthy and Tromba have also treated Fredholm maps $F$ with positive index. The corresponding degree is no longer an integer but a Pontrjagin framed cobordism class (see [27], [28], [46], [12], [11] which also contain further references). This is however difficult to work with. In some situations one may use stable homotopy or cohomotopy; in [46] some application to elliptic boundary value problems has been made. But so far no natural example has arisen in which these methods have been used. I am confident that some will occur.

To conclude the discussion of topological methods for (1), it should be mentioned that many mathematicians have made important contributions in this field. To mention a few: M. A. Krasnoselskii, F. Browder, R. Nussbaum, W. Petryshyn, and for more general use of homotopy theory K. Geba and A. Granas. See [11], [31], [47] for some useful references to recent developments.

I.5 In the study of periodic solutions of systems of ordinary differential equations much work has been done to find invariants associated with closed orbits, or isolated families of closed orbits, i.e., which are invariant under perturbation of the system. This is to guarantee that the perturbed equations also possess closed orbits near the original ones.

Let us recall the simplest situation. Consider a system of ordinary differential equations for a vector $x$ in $R^{n}$.

$$
\dot{x}=\frac{d x}{d t}=f(x) .
$$

Suppose we have a nonconstant closed (i.e. periodic) orbit $x_{0}(t)$. Through a point $y_{0}$ on the orbit consider a hyperplane $P$ perpendicular to the orbit at $y_{0}$. On it, near $y_{0}$ consider the Poincaré map $F$ of $P \rightarrow P$ defined as follows. From any point $y \in P$ near $y_{0}$ follow the solution curve of the system until it strikes $P$ again for the first time near $y_{0}-$ it is easily seen that it must. Let $z$ be the point where it first strikes $P$. The map $y \mapsto z$ is called the Poincare map. Clearly $y_{0}$ is a fixed point of this map.

Suppose $y_{0}$ is an isolated fixed point, set $F(y)=y-z(y)$. Then for a small ball $B$ in $P$ centered at $y_{0}$ the degree

$$
\operatorname{deg}(F, B, 0)=\nu
$$

is well defined. If $\nu \neq 0$ we may then infer that any slight perturbation of the system (4) has a periodic solution lying close to $x_{0}(t)$ (and in fact its period will be close to that of $\left.x_{0}(t)\right)$.

In [30] Fuller introduced a more general notion, now called the Fuller index, attached to a closed orbit of (4). This has proved very useful; Mallet-Paret and Yorke [40] modified this to study global connected sets of orbits in case $f$ depends on a parameter. The reader may also find many useful references here.

Conley [21] has introduced a very interesting generalization of the Morse index for any isolated set which is invariant under the flow of (4), i.e. the maximal invariant set in some neighbourhood of itself. In Morse theory, one studies Morse functions: real functions $f$ on $n$-dimensional manifolds having 
nondegenerate stationary points, i.e. at a stationary point the Hessian matrix of second derivatives

$$
f_{x x} \text { is nonsingular. }
$$

The number $k$ of negative eigenvalues of the Hessian is called the index of the stationary point. The global structure of the (compact) manifold is tied to the number of critical points of $f$ with various indices.

Conley's generalized Morse index assigned to a compact isolated invariant set $S$ is the homotopy type of a pointed topological space, i.e. a space with a distinguished point in it. Very roughly, it is defined in the following way. Let $N$ be a neighbourhood containing $S$ compactly in its interior. An index pair $\left\langle N_{1}, N_{2}\right\rangle$ is a pair $N_{1}, N_{2}$ of compact subsets of $N$ satisfying several conditions:

(i) relative to $N$ they are invariant under forward flow i.e. for $i=1,2$, $\forall x_{0} \in N_{i}$ and $\forall t>0$, the point $x\left(t, x_{0}\right)$ (i.e. the point on the orbit starting at $x_{0}$, after time $t$ has elapsed) is either in $N_{i}$ or is not in $N$,

(ii) $S \subset \operatorname{int}\left[N_{1} \backslash\left(N_{1} \cap N_{2}\right)\right]$,

(iii) $N_{2}$ is roughly the set of points through which orbits eventually leave $N$ under the flow, i.e. if some point $x$ in $N_{1}$ eventually (its orbit) flows out of $N$ then it first passes through $N_{2}$.

The generalized Morse index of $S$ is the homotopy class

$$
h(S)=\left[N_{1} /\left(N_{1} \cap N_{2}\right)\right] .
$$

(Recall that two spaces $A$ and $B$ are homotopy equivalent if there exist two continuous mappings $\phi: A \rightarrow B$ and $\psi: B \rightarrow A$ such that $\phi \circ \psi$ and $\psi \circ \phi$ are homotopic to the identity in $B$ and $A$ respectively.)

One of the main properties is

THEOREM. $h(S)$ is independent of the choice of $N_{1}$ and $N_{2}$ and also of the neighbourhood $S$. Furthermore suppose $N \subset N^{\prime}$, and for $N^{\prime}$ we have an index pair $\left\langle N_{1}^{\prime}, N_{2}^{\prime}\right\rangle$, and suppose

$$
\left[N_{1} /\left(N_{1} \cap N_{2}\right)\right] \neq\left[N_{1}^{\prime} /\left(N_{1}^{\prime} \cap N_{2}^{\prime}\right)\right] \text {. }
$$

Then $N^{\prime}$ must contain some other invariant set besides $S$.

Recently Amann and Zehnder [4] have applied this theorem to prove the existence of solutions of some nonlinear partial differential equations. They use the result in a rather special situation-which we now describe in the simplest case. Consider a real smooth function $f$ defined on all of $R^{n}$, which, near the origin, is equal to a nondegenerate (i.e. having no zero eigenvalues) homogeneous quadratic having $k$ negative eigenvalues. Near infinity $f$ is also assumed to be a nondegenerate homogeneous quadratic having $k^{\prime}$ negative eigenvalues.

LEMMA. If $k^{\prime} \neq k$ then $f$ must have a nontrivial critical point.

REMARK. In case $k^{\prime}-k$ is odd the result is easily proved using degree theory. Set $F=\operatorname{grad} f$. The local degree of $F$ near the origin,

$$
\operatorname{deg}(F,|x|<\varepsilon, 0)=(-1)^{k}
$$


as is easily seen. On the other hand for $R$ large

$$
\operatorname{deg}(F,|x|<R, 0)=(-1)^{k^{\prime}} .
$$

Thus if these numbers are different, $F$ has to vanish at some point on $\varepsilon<|x|<R$. This proof does not require that $F$ be a gradient map in $\varepsilon<|x|<R$. On the other hand if $F$ is not a gradient map and $k^{\prime}-k$ is even, the result need not hold. This argument also shows that if we just assume the condition on $f$ near infinity, then it follows that $f$ must have a stationary point.

Let us see how the Conley index is used to prove the lemma. We may suppose that near the origin,

$$
f=\sum_{1}^{n-k} x_{j}^{2}-\sum_{\alpha>n-k} x_{\alpha}^{2} .
$$

Consider the gradient flow $\dot{x}=\operatorname{grad} f$. The origin is an isolated invariant set $S$. Let us take $N=\{|x|<R\}, k$ large, $N_{1}=|x|<\varepsilon$ small and the set generated by its flow in $N$, and $N_{2}=N_{1} \cap\{R-1 \leqslant|x|<R\}$. In this case one finds

$$
\left[N_{1} /\left(N_{1} \cap N_{2}\right)\right]=\left[S^{n-k}, x_{0}\right]
$$

where $x_{0}$ is a fixed point on $S^{n-k}$.

On the other hand if we take $N_{1}^{\prime}$ to be a large ball $|x|<R / 2$ and the set generated by its flow in $N$ and take $N_{2}^{\prime}$ correspondingly, using the behaviour near infinity we find

$$
\left[N_{1}^{\prime} /\left(N_{1}^{\prime} \cap N_{2}^{\prime}\right)\right]=\left[S^{n-k^{\prime}}, x_{0}\right] \text {. }
$$

Since the homotopy classes are different, for $k \neq k^{\prime}$, we conclude from Conley's theorem that the region

$$
\varepsilon<|x|<R
$$

must contain another invariant set under the gradient flow. However, since it is a gradient flow, we see that $f$ increases on a flow line. It follows easily that this other invariant set must consist of invariant points, i.e. stationary points of $f$.

K. C. Chang [19] has found a proof of the lemma which uses only Morse theory. It makes use of the Morse inequalities for a Morse function defined on a domain with boundary, and having suitable behaviour on the boundary.

We are coming closer to our second topic, variational methods, concerned with finding stationary points of real functions. To conclude this section, we mention one more useful result which is due to Castro and Lazer [17]. Though the result seems intuitively clear, I do not know an elementary proof of it.

THeOREM. Let $f$ be a smooth function defined on $R^{n}$ having only a finite number of critical points and such that $f(x) \rightarrow+\infty$ as $|x| \rightarrow \infty$. Then for $R$ large

$$
\operatorname{deg}\left(\operatorname{grad} f, B_{R}, 0\right)=1 \text {. }
$$


They use this to prove the existence of more than one critical point:

Lemma. Let $f$ be a smooth function in $R^{n}$ with $f(x) \rightarrow+\infty$ as $|x| \rightarrow \infty$. Assume the origin is a nondegenerate critical point and that $f$ achieves its minimum at a point $x_{0} \neq 0$. Then $f$ has at least three stationary points.

This follows easily from the preceding theorem, using degree theory.

\section{Variational methods}

In the 1960's classical Morse theory was extended to real functions defined on infinite-dimensional spaces, such as Banach manifolds, by Palais, Smale and others-including the Morse inequalities. This material can be found in several sources, see for example [57], [49] and [50], and will not be taken up in these lectures.

We will discuss arguments for proving the existence of stationary points of real functions $f$ defined on a Banach space $X$. In case the function $f$ is bounded from above or below it is reasonable to try to show that it attains a maximum or minimum. For convex functions, a classical result in this direction is

THEOREM. Let $f$ be a lower-semi-continuous convex function, bounded from below, defined on a reflexive Banach space, such that $f(x) \rightarrow \infty$ as $x \rightarrow \infty$. Then $f$ attains its minimum.

Results on existence of stationary points make use of some kind of compactness. In the theorem just quoted the compactness is hidden. The proof uses the fact that a closed bounded convex set in a reflexive Banach space is compact in the weak topology. Here is the proof of the theorem. Let $x_{n}$ be a minimizing sequence, i.e. $f\left(x_{n}\right) \rightarrow \inf f$. Clearly the $\left|x_{n}\right|$ are bounded, and so a subsequence which we still denote by $x_{n}$ converges weakly to some $x$. By Mazur's lemma there is a sequence of convex combinations $y_{i}$ of $\left(x_{1}, \ldots, x_{i}\right), i=1,2, \ldots$, converging to $x$ strongly. From the convexity of $f$ we see that $f\left(y_{i}\right) \rightarrow \inf f$, and from the lower-semi-continuity we conclude that $f(x)=\inf f$. See [25] for variational problems for convex functions.

If $f$ is not convex it need not achieve its infimum. However the following result of Ekeland [23] shows the existence of points which are almost points of minimum.

THEOREM. Let $X$ be a complete metric space and $f$ a real lower semicontinuous function defined on $X$ and bounded from below. Then there exists some $x_{0}$ in $X$ such that

$$
f(x)>f\left(x_{0}\right)-\operatorname{dist}\left(x, x_{0}\right) \text { for all } x \in X, x \neq x_{0} .
$$

II.1 A compactness-kind of condition that is usually employed in proving the existence of stationary points is the condition $\mathrm{C}$ of Palais and Smale, now called the (PS) condition, for a function $f \in C^{1}$.

(PS) Any sequence $\left\{x_{j}\right\} \in X$ such that $\left|f\left(x_{j}\right)\right| \leqslant M$ and $f^{\prime}\left(x_{j}\right)$ $\rightarrow 0$ in norm in $X^{*}$ (the dual space) has a strongly convergent subsequence. 
Here $f^{\prime}(x)$ represents the derivative of $f$ at $x$, and is an element of the dual space $X^{*}$ of continuous linear functionals on $X$. Such a function always takes on its infimum:

LEMMA. Let $f$ be a real $C^{1}$ function on a Banach space satisfing (PS) and bounded from below. Then $f$ achieves a minimum at some point.

REMARKS. 1. In practice, given a function $f$ for which we seek solutions of

$$
F(x) \equiv f^{\prime}(x)=0,
$$

the exact topological space on which one should work is not given to us. It should be chosen sufficiently small so that $f$ is smooth, say in $C^{1}$. On the other hand it cannot be taken too small for then the condition (PS) will not hold. One has to strike just the right balance.

2. It is natural to ask how verification of (PS) differs from the usual task of obtaining a priori estimates, i.e., showing that the map $F(x)=f^{\prime}(x)$ is proper. There is one additional useful item here; the fact that the $f\left(x_{j}\right)$ are all bounded.

Let us illustrate these remarks by discussing a simple semilinear boundary value problem for a real function $u(x)$ defined in a bounded domain $G$ in $R^{n}$ with smooth boundary $\partial G$.

$$
\Delta u+g(x, u)=0 \text { in } G, \quad u=0 \text { on } \partial G ;
$$

(here $\Delta=\Sigma \partial^{2} / \partial x_{i}^{2}$ is the Laplace operator). We seek a positive solution $u$ assuming

$$
\left\{\begin{array}{l}
g \text { is smooth and } g(x, u)>0 \text { for } u>0, x \in \Omega, \\
g(x, u)=o(u) \text { as } u \rightarrow 0 \text { uniformly in } x, \\
g(x, u)=a(x) u^{\sigma}, a(x)>0 \text { in } \bar{G}, \sigma>1, \text { for } u \text { large. }
\end{array}\right.
$$

If $\sigma<(n+2) /(n-2)$, (5) has a positive solution $u(x)$-as we will see. The solution will be obtained as a stationary point of the functional

$$
f(u)=\int_{G}\left[\frac{1}{2}|\operatorname{grad} u|^{2}-G(x, u(x))\right] d x
$$

where $G(x, u)=\int_{0}^{u} g(x, s) d s$. Since we are looking for a positive solution there is no loss of generality in changing $g$ for $u<0$ and assuming that $g(x, u)>0$ for $u=0$ and

$$
g(u)=a(x)|u|^{\sigma} \quad \text { for }|u| \text { large. }
$$

For such a $g$, any nontrivial solution of (5) will automatically be positive, by the maximum principle, and so a solution of our original equation.

What space of functions should we work in? and will condition (PS) hold?

We wish $f$ to be of class $C^{1}$ in the space. From the form of $f$ it seems reasonable to work in a space contained in $H_{0}^{1}$, i.e. the space of functions in the Sobolev space $H^{1}$ which vanish on the boundary $\partial G$. According to the 
Sobolev embedding theorem,

$$
\left\{\begin{array}{l}
\text { the injection map } H_{0}^{1} \rightarrow L^{p}(G), p>1, \text { is } \\
\text { continuous } \Leftrightarrow p \leqslant \frac{2 n}{n-2}, \text { and compact } \Leftrightarrow p<\frac{2 n}{n-2} .
\end{array}\right.
$$

Now

$$
G(x, u) \sim \frac{a(x) u^{\sigma+1}}{\sigma+1} \text { for } u \text { large, }
$$

so for $f$ to be well defined on $H_{0}^{1}$ we should require $\sigma+1 \leqslant 2 n /(n-2)$, i.e. $\sigma \leqslant(n+2) /(n-2)=\sigma_{0}$. In case $\sigma>\sigma_{0}$, one might be tempted to work in, say, $H^{s} \cap H_{0}^{1}$ for some $s>1$. But after a little reflection, one concludes that control on $f$ cannot yield control on the $H^{s}$ norm, so that (PS) cannot hold. Thus it seems sensible to work in the space $X=H_{0}^{1}$ and to require $\sigma \leqslant \sigma_{0}$. We may then take $\|u\|_{H_{0}^{1}}^{2}=\int_{G}|\operatorname{grad} u|^{2}$. But then does (PS) hold?

LemMa. Condition (PS) holds $\Leftrightarrow \sigma<\sigma_{0}$.

Sketch of Proof of $\Leftarrow$. We leave the other part as an exercise. Let $\left\{u_{j}\right\}$ be a sequence in $H_{0}^{1}$ such that

$$
\left|f\left(u_{j}\right)\right| \leqslant M, \quad f^{\prime}\left(u_{j}\right) \rightarrow 0 \quad \text { in norm. }
$$

The last condition means that

$$
\int_{\Omega} \operatorname{grad} u_{j} \operatorname{grad} \zeta-g\left(x, u_{j}\right) \zeta=o(1) \cdot\|\zeta\|_{H_{0}^{1}}, \forall \zeta \in H_{0}^{1} .
$$

Inserting $\zeta=u_{j}$ we find, setting $\left\|u_{j}\right\|_{H_{0}^{1}}=c_{j}$,

$$
\frac{1}{c_{j}} \int_{G}\left|\operatorname{grad} u_{j}\right|^{2}-u_{j} g\left(x, u_{j}\right) \rightarrow 0 .
$$

Since $\int_{G} \frac{1}{2}\left|\operatorname{grad} u_{j}\right|^{2}-G\left(x, u_{j}\right)$ is bounded and

$$
\int G\left(x, u_{j}\right)-\frac{1}{\sigma+1} u_{j} g\left(x, u_{j}\right) \text { is bounded }
$$

we find

$$
\int \frac{\sigma+1}{2}\left|\operatorname{grad} u_{j}\right|^{2}-u_{j} g\left(x, u_{j}\right) \text { is bounded. }
$$

Claim. The norms $c_{j}=\left\|u_{j}\right\|_{H_{0}^{1}}$ are bounded. If not, for a suitable subsequence, we may suppose $c_{j} \rightarrow \infty$. Combining (10) and (11) we find

$$
\left(\frac{\sigma+1}{2}-1\right) c_{j} \text { is bounded }
$$

-a contradiction since $\sigma>1$; the claim is proved.

We may select a subsequence, still denoted by $u_{j}$, which converges weakly to $u$ in $X$, and converges strongly to $u$ in $L^{\beta}$, for some $\beta$ in $\sigma+1<\beta<\sigma_{0}+$ 1. This is possible in view of the Sobolev embedding theorem quoted above. One then finds easily that $g\left(x, u_{j}(x)\right)$ converges to $g(x, u(x))$ in $L^{\sigma_{0}+1}$. It 
follows easily, considering $(9)_{j}-(9)_{k}$, with $\zeta=u_{j}-u_{k}$, that

$$
\left\|u_{j}-u_{k}\right\|_{H_{0}^{1}}^{2} \rightarrow 0 \text { as } j, k \rightarrow \infty
$$

and the lemma is proved.

So for $\sigma<(n+2) /(n-2)$, our functional $f$ given in (7) satisfies (PS). Next we will see how one finds nontrivial stationary points.

II.2 We shall present several arguments for finding stationary points of a functional $f$ which is unbounded from above and below-leading us to look for saddle points. These are found by some min max argument. One considers a certain class $A$ of sets $\Sigma$ and forms

$$
\max _{u \in \Sigma} f(u)
$$

for any $\Sigma$ in $A$ and then

$$
c=\inf _{\Sigma \in A} \max _{u \in \Sigma} f(u),
$$

and tries to show that the number $c$ is a stationary value of $f$, i.e., is a value of $f$ at some stationary point.

In case $f$ is an even function, $f(u)=f(-u)$, there is the well-developed category theory of Liusternik-Schnirelman and the related notion of genus to find solutions (often infinitely many). In this approach one takes for $A$ the class of all sets $\Sigma$ of category $\geqslant$ some number. An excellent presentation of this theory may be found in [50], and we will not discuss this method. Analogous theories in case other group actions are present have been developed by various authors (see Benci [8], [9]).

The methods we will take up here are all variations on a basic result known to everyone who has done any walking in the hills: the mountain pass lemma.

Let us first describe this for a real function $f(x)$, defined on the plane-representing the height of the land above sea level over the point $x \in R^{2}$. Suppose the origin lies in a valley surrounded by a ring of mountains, i.e. there is an open neighbourhood $\Omega$ of the origin such that

$$
f(x) \geqslant c_{0}>f(0) \text { for } x \in \partial \Omega .
$$

Suppose that there is some point $x_{0}$ outside, i.e., $x_{0} \notin \bar{\Omega}$, such that $f\left(x_{0}\right)<c_{0}$. We wish to walk from $x_{0}$ to 0 climbing as little as possible, i.e. keeping $f$ as low as possible. Naturally the way to do this is to take a path crossing the mountain over the lowest mountain pass. The top of the mountain pass corresponds to a stationary point of $f$ and there the value of $f$, i.e. the stationary value, equals

$$
c=\inf _{P} \max _{x \in P} f(x) \geqslant c_{0} .
$$

Here $P$ represents any continuous path from $x_{0}$ to 0 and inf is taken with respect to all such paths. Clearly every such path has to intersect $\partial \Omega$, and so $\max _{P} f \geqslant c_{0}$. This is the assertion of the mountain pass lemma-that the number $c$ so defined is a stationary value of $f$. Note that $c$ will, in general, be less than $\sup f$.

This formulation is not quite correct since the plane is not compact, and one should add a compactness condition, for example, (PS). The lemma is 
then true and holds even in Banach space. In this form it is due to Ambrosetti and Rabinowitz [6], though similar arguments had been employed many years ago in proving the existence, in special situations, of several minimal surfaces spanning some curve.

Mountain Pass Lemma [6]. Let $f$ be a $C^{1}$ real function defined on a Banach space $X$ and satisfying (PS). Assume there is an open neighbourhood $\Omega$ of 0 and a point $x_{0} \notin \bar{\Omega}$ such that

$$
f(0), f\left(x_{0}\right)<c_{0} \leqslant \inf _{\partial \Omega} f .
$$

Then the following number is a critical value of $f$,

$$
c=\inf _{P} \max _{P} f \geqslant c_{0}
$$

where $P$ represents any continuous path from $x_{0}$ to 0 .

In recent years extensions and variations of this lemma have proved very useful and we will present several in the next section. But first we show, with its aid, how to obtain a positive solution of (5) for $\sigma<\sigma_{0}=(n+2) /(n-2)$.

THEOREM. For $g$ satisfying (6) with $\sigma<\sigma_{0}$ there exists a positive solution of (5).

Proof. Consider the functional (7) in $X=H_{0}^{1}$. We have shown that it satisfies (PS) and, for $g$ smooth, one verifies that it is of class $C^{1}$. Note that $f(0)=0$. To find a nontrivial solution via the mountain pass lemma we will show that for some $r>0, c_{0}>0$ we have

$$
f(u) \geqslant c_{0} \text { if }\|u\|_{H_{0}^{1}}=r .
$$

Furthermore for some $u_{0},\left\|u_{0}\right\|_{H_{0}^{1}}>r, f\left(u_{0}\right) \leqslant 0$. From the mountain pass lemma it will follow that there is a stationary point $u \in H_{0}^{1}$ of $f$, with $f(u) \geqslant c_{0}>0$. Thus $u \neq 0$.

The function $u$ is a generalized solution of (5) but by a standard boot strap argument one shows that it is smooth, and then, by the maximum principle, is positive.

To prove (11) we now make use of (6) from which we may infer that for any $\varepsilon>0$

$$
G(x, u) \leqslant \varepsilon|u|^{2}+c_{\varepsilon}|u|^{\sigma+1}
$$

Thus for $\|u\|_{H_{0}^{1}}=r$,

$$
\begin{aligned}
\int G(x, u(x)) & \leqslant \int \varepsilon u^{2}+C_{\varepsilon}|u|^{\sigma+1} \\
& \leqslant C\left(\varepsilon r^{2}+C_{\varepsilon} r^{\sigma+1}\right), \quad C \text { independent of } \varepsilon,
\end{aligned}
$$

by (8). Consequently for $\|u\|_{H_{0}^{1}}=r$,

$$
f(u)=\frac{1}{2} r^{2}-\int G(x, u) \geqslant \frac{1}{2} r^{2}-C\left(\varepsilon r^{2}+C_{e} r^{\sigma+1}\right)
$$

and (12) follows for $\varepsilon$ and then $r$, chosen small. 
Finally we have to choose $u_{0}$ so that

$$
\left\|u_{0}\right\|_{H_{0}^{1}}>r \text { and } f\left(u_{0}\right) \leqslant 0
$$

Simply take $u_{0}=\lambda v$, for $v$ a fixed smooth positive function vanishing on the boundary, and $\lambda$ sufficiently large. The existence theorem is proved.

ReMARKS. Suppose we consider in place of (5) the boundary value problem

$$
L u+g(x, u)=0 \text { in } G, \quad u=0 \text { on } G,
$$

with $g$ satisfying (6) and $L$ a linear elliptic operator with smooth coefficients

$$
L u=a^{i j}(x) u_{x_{i} x_{j}}+a^{i}(x) u_{x_{i}}+a(x) u,
$$

with $a^{i j}(x) \xi_{i} \xi_{j}$ positive definite, and $a(x) \leqslant 0$. If $L$ is not formally selfadjoint the problem cannot be formulated as a variational one. In [13] Brézis and Turner solved this (permitting $g$ to depend also on $\operatorname{grad} u$ ) in case $\sigma<$ $n /(n-2)$. In this case they were able to obtain the appropriate a priori estimates for all positive solutions of $\left(5^{\prime}\right)$. In case $L=\Delta$ the corresponding a priori estimates for the general case $\sigma<(n+2) /(n-2)$ have recently been established by De Figueiredo, Lions and Nussbaum [22], and for general $L$ by Gidas and Spruck [32].

For $\sigma=\sigma_{0}=(n+2) /(n-2)$ a nonzero solution of (5) need not exist, as was shown by Pokhozhaev for $g=u^{\sigma_{0}}$ in a star-shaped domain (see [32]). On the other hand in a ring shaped domain $r_{1}<|x|<r_{2}$, and $g=u^{\sigma_{0}}$, there is a positive solution.

Several problems of current interest are just on the borderline where (PS) fails. Among these are the Yang-Mills equations of Physics, and the Yamabe problem in Differential Geometry. In the latter, one is given a compact Riemannian manifold without boundary and wishes to find a new metric on it which is conformally equivalent to the given one, and having constant scalar curvature.

II.3 We now take up some extensions of the mountain pass lemma. The following is due to Rabinowitz [52]; we shall present a more general form due to $\mathrm{Ni}$ [45]. Let us first describe it for a function $f$ defined in $R^{3}$, of class $C^{1}$ and satisfying (PS).

Generalization. Assume there are two disjoint simple closed curves $\Gamma_{0}, \Gamma_{1}$ in $R^{3}$ which link each other and such that the values of $f$ on one are bounded away from its values on the other, i.e.,

$$
f(x) \leqslant c_{0}<c_{1} \leqslant f(y), \quad \forall x \in \Gamma_{0}, \forall y \in \Gamma_{1} .
$$

Then the following number is a critical value of $f$.

$$
c=\inf _{\Sigma} \max _{x \in \Sigma} f(x) \geqslant c_{1}
$$

where $\Sigma$ represents any surface spanning the curve $\Gamma_{0}$ and inf is taken over all such $\Sigma$.

The linking of the two curves is simply to ensure that any surface $\Sigma$ spanning $\Gamma_{0}$ necessarily intersects $\Gamma_{1}$, so that $\max _{\Sigma} f \geqslant c_{1}$. This result seems much less intuitive than the preceding. Here is the general form. 
Generalized MPL. Let $f$ be a $C^{1}$ real function defined on $R^{N}$ satisfying (PS). Let $\phi$ and $\psi$ be continuous mappings of spheres $S^{k}$ and $S^{N-k-1}$ into $R^{N}$ whose images are disjoint and have nontrivial linking. Assume that

$$
f(\phi(x)) \leqslant c_{0}<c_{1} \leqslant f(\psi(y)), \quad \forall x \in S^{k}, \forall y \in S^{N-k-1} .
$$

Then

$$
c=\inf _{h} \max _{x \in B^{k+1}} f(h(x)) \geqslant c_{1}
$$

is a critical value of $f$. Here $h$ represents any continuous extension of $\phi$ inside the unit ball $B^{k+1}$ in $R^{k+1}$, and the inf is taken over all such $h$.

REMARKs. If we reverse the roles of $\phi$ and $\psi$ we see that there is also a critical value $\leqslant c_{0}$. The condition on linking is simply to ensure that for every extension $h, h\left(B^{k+1}\right)$ necessarily intersects the image $\psi\left(S^{N-k-1}\right)$. In fact the theorem holds if the set $\psi\left(S^{N-k-1}\right)$, on which $f$ is assumed to be $\geqslant c_{1}$, is any set with the property that it intersects every image $h\left(B^{k+1}\right)$. Furthermore, in place of $R^{N}$ we may have an $N$-dimensional manifold, and in place of $S^{k}$, the boundary of a compact $(k+1)$-dimensional manifold. The original mountain pass lemma corresponds essentially to the case $k=0$. See [45] for an infinite-dimensional version and [54], [10] and [8] for related results.

Here is still another variant of these ideas due to A. Castro [16].

TheOREM. Let $f$ be a $C^{1}$ real function defined on a Banach space $X$ satisfying (PS). Assume $X$ has a direct sum decomposition

$$
X=X_{1} \oplus X_{2}, \quad \operatorname{dim} X_{1}=k<\infty .
$$

Let $S_{1}\left(B_{1}\right)$ be the unit sphere (ball) in $X_{1}$, and $S_{2}$ a sphere $\|x\|=R$ in $X_{2}$. Assume for some constants $\beta<\alpha ; c_{0}<c_{1}$,

$$
\begin{array}{ll}
f(x) \leqslant c_{0} & \text { for } x \in X_{1},\|x\|<1, \\
f(x) \leqslant \beta & \text { for } x \in X_{1},\|x\|=1 \text { i.e. } x \in S_{1}, \\
f(x) \geqslant \alpha & \text { for } x \in X_{2},\|x\| \leqslant R, \\
f(x) \geqslant c_{1} & \text { for } x \in X_{2},\|x\|=R \text { i.e. } x \in S_{2} .
\end{array}
$$

Then the following is a critical value of $f$ :

$$
c=\inf _{\Sigma} \max _{x \in \Sigma} f(x) \geqslant \alpha .
$$

Here $\Sigma$ is any $k$-dimensional surface in $X$ spanning $S_{1}$ and homotopic to $B_{1}$ in $X \backslash S_{2}$, and the inf is taken with respect to all such $\Sigma$.

The mountain pass lemma and all the extensions given here, as well as the lemma near the beginning of $\$ I I .2$, are proved in the same way by a well-known procedure which is presented in a systematic way in Palais [49]. We will indicate it by sketching the proof of the Generalized MPL. To avoid some technicalities let us consider the case that $X$ is a Hilbert space and $f \in C^{2}$.

Proof. Assume that the number $c$ defined there is not a critical value. 
Then for $0<\varepsilon$ small, it follows from (PS) that for some $\alpha>0$

$$
\left\|f^{\prime}(x)\right\| \geqslant \alpha>0 \text { if } c-\varepsilon<f(x)<c+\varepsilon .
$$

We may take $\varepsilon<c_{1}-c_{0}$. Choose an extension $h$ of $\phi$ inside $B^{k+1}$ with

$$
f(h(x)) \leqslant c+\frac{\varepsilon}{2}, \quad \forall x \in B^{k+1} .
$$

We shall make use of a modification of the gradient flow associated to $-f$. Let $\eta(u)$ be a locally Lipschitz function satisfying

$$
\begin{array}{ll}
\eta \equiv 1 \quad \text { on } f^{-1}\left(c-\frac{\varepsilon}{2}, c+\frac{\varepsilon}{2}\right), \\
\eta \equiv 0 \quad \text { outside } f^{-1}(c-\varepsilon, c+\varepsilon) .
\end{array}
$$

Consider the flow associated with the differential equation in $X$ :

$$
\frac{d x}{d t}=-\eta(x) \frac{f^{\prime}(x)}{\left\|f^{\prime}(x)\right\|^{2}}, \quad x(0)=y,
$$

and denote the solution, which exists locally, by $x(t)=x(t, y)$. Note that on a solution curve $x(t)$ we have

$$
\frac{d}{d t} f(x(t))=-\eta(x(t)) \leqslant 0 .
$$

Thus within a time interval $\varepsilon$, every point $x$ in the region

$$
f^{-1}(c-\varepsilon / 2, c+\varepsilon / 2)
$$

where $\eta \equiv 1$, flows into the region where $f \leqslant c-\varepsilon / 2$. In particular, since $f$ decreases under the flow, it follows that the image

$$
\tilde{h}(y)=x(\varepsilon, h(y)), \quad y \in B^{k+1},
$$

lies in the region where $f \leqslant c-\varepsilon / 2$. However, for $y \in S^{k}=\partial B^{k+1}$, we have $f(\phi(y)) \leqslant c_{0}<c_{1}-\varepsilon \leqslant c-\varepsilon$. Hence $\eta(y)=0$ and the point $y$ does not move under the flow. Consequently

$$
\tilde{h}(y)=h(y)=\phi(y) \text { for } y \in S^{k},
$$

and $\tilde{h}$ is an admissible map in our competition. But then $\tilde{h}\left(B^{k+1}\right)$ cannot lie in the region where $f \leqslant c-\varepsilon / 2$. Contradiction!

II.4 This section will be devoted to two results of Rabinowitz [53] on periodic solutions for a Hamiltonian system-as illustration of the use of some of the results in the preceding section.

A Hamiltonian system is a system of ordinary differential equations for a pair of vectors $p, q$ in $R^{n}$, depending on time

$$
\begin{gathered}
\dot{p}=\frac{d p}{d t}=-H_{q}, \quad \text { i.e., } \frac{d p^{i}}{d t}=-\frac{\partial H}{\partial q^{i}}, \\
\dot{q}=\frac{d q}{d t}=H_{p}, \quad \text { i.e., } \frac{d q^{i}}{d t}=\frac{\partial H}{\partial p^{i}} .
\end{gathered}
$$

Here the Hamiltonian $H(p, q)$ is a given real function defined on $R^{2 n}$. The 
problem is to prove the existence of nontrivial, i.e. nonconstant, time periodic solutions.

It is trivial to verify that on any solution curve $p(t), q(t)$, the Hamiltonian $H(p(t), q(t))$ is constant. Thus it makes sense to look for a periodic solution on a given level surface of $H$. The first result of Rabinowitz is the following (for convenience we take $H$ to be smooth).

Theorem A. Assume that $\operatorname{grad} H \neq 0$ on the level set $\Sigma$ where $H=1$ (so that $\Sigma$ is a regular hypersurface). Assume that $\Sigma$ is compact and strictly star shaped about the origin, i.e., any ray from the origin hits $\Sigma$ at just one point and nontangentially. Then there is a nontrivial periodic solution of (13) on $\Sigma$.

This is a beautiful result and we will give, essentially, a complete proof of it. There are earlier perturbation results about the existence of nontrivial periodic solutions near a trivial one, and also a global result by Weinstein in case $H$ is convex-by quite different methods. (See [53] for references.) It is natural to ask whether the result is true if $\Sigma$ is merely diffeomorphic to a sphere. This problem is open.

First a useful

REMARK. If $\operatorname{grad} H \neq 0$ on a level set $\Sigma$ of $H$, then the set of integral curves of (13) on $\Sigma$ depend only on the hypersurface $\Sigma$-they are otherwise independent of $H$.

This is easily proved. Suppose $H^{\prime}$ is another Hamiltonian having $\Sigma$ as level set and with grad $H^{\prime} \neq 0$ on $\Sigma$. Then

$$
\operatorname{grad} H^{\prime}=f \operatorname{grad} H \text { on } \Sigma \text {, }
$$

with $f$ a positive function on $\Sigma$. Suppose $p(t), q(t)$ is an integral curve for $H^{\prime}$ on $\Sigma$, i.e.

$$
\dot{p}=-H_{q}^{\prime}=-f H_{q}, \quad \dot{q}=H_{p}^{\prime}=f H_{p} .
$$

If we reparametrize this curve by a new variable $s$ with $d s=f d t$, we see that

$$
d p / d s=-H_{q}, \quad d q / d s=H_{p}
$$

on the curve, and the remark is proved.

Theorem $\mathbf{A}$ is a simple consequence of a very general result in [53]:

THEOREM B. Consider a $C^{1}$ Hamiltonian $H$ satisfying

(i) $H \geqslant 0$ and $H(p, q)=o\left(|p|^{2}+|q|^{2}\right)$ near the origin,

(ii) $0<H<\theta\left(p \cdot H_{p}+q \cdot H_{q}\right), 0<\theta<\frac{1}{2}$ for $|p|^{2}+|q|^{2}$ large.

Then for this $H$, and any given $T>0$, there exists a nontrivial periodic solution of (13) with period $T$.

REMARK. By integrating condition (ii) we find

$$
H \geqslant C\left(|p|^{2}+|q|^{2}\right)^{1 / 2 \theta} \text { near infinity, } C>0,
$$

i.e. $H$ grows faster than quadratically near infinity.

Derivation of Theorem A. By the remark, we may replace the given Hamiltonian by any other provided the set where it equals 1 is $\Sigma$. Choose such a new $H$ to be positive homogeneous of degree 4. The existence of such 
an $H$ follows immediately from the strict star-shaped property, and in fact it is only here that the property is used.

Applying Theorem B with $T=1$ to this new $H$ we infer that there is a nontrivial solution $p(t), q(t)$ with period 1 . It lies on some level surface $H=c$, but not necessarily the desired one $\Sigma: c=1$. To obtain such a solution we simply perform a suitable scaling (stretching) using the homogeneity of $H$, and the theorem is proved.

We are going to sketch some of the main points in the proof of Theorem B. By a stretching of the time variable, and of $H$, we may suppose that $T=2 \pi$. In the space of $2 \pi$ periodic functions $p(t), q(t)$, consider the functional

$$
F(p, q)=\int_{0}^{2 \pi}\left[p \cdot \frac{d q}{d t}-H(p(t), q(t))\right] d t .
$$

It is readily verified that a stationary point (a pair of $2 \pi$ periodic functions $p(t), q(t))$ of $F$ is a solution of (13). That is, (13) is the corresponding Euler equation. The pair $p(t) \equiv q(t) \equiv 0$ is such a trivial solution but we seek a nontrivial one. Since $H$ grows faster than quadratically at infinity we see, on taking $p=q=$ large constant vectors, that $F$ is unbounded from below. Taking bounded and highly oscillatory functions $p, q$, we see that $F$ is also unbounded from above. We wish, therefore, to find a saddle point of $F$-other than the trivial one.

The procedure in [53] is the following: (a) approximate the space by a suitable finite-dimensional one; (b) in the finite-dimensional space, prove the existence of a nontrivial stationary point; (c) go to the limit in the approximation. The last part is rather technical. In particular, in going to the limit, one has to make sure that the stationary point obtained in step (b) does not run off to infinity nor to the origin.

The finite-dimensional approximation is the following: Let us consider the space of $2 \pi$-periodic functions $(p(t), q(t))$ in $L^{2}$. Then the action integral

$$
Q(p, q)=\int^{2 \pi} p \cdot \frac{d q}{d t} d t
$$

is a quadratic form and associated with it are its eigenvalues $\lambda$, which are integers, and eigenvectors spanned by finite Fourier series. Take as approximating space, the space $R^{N}$ spanned by the eigenfunctions associated with the eigenvalues in $|\lambda| \leqslant M$ for large $M$. Restricting $F$ to this space we will show how to obtain a nontrivial stationary point-using just some simple properties of $F$. First decompose $R^{N}=E_{1} \oplus E_{2}$ where $E_{1}$ is the subspace spanned by all eigenvectors of $Q$ having nonpositive eigenvalues, $E_{2}$ is the subspace spanned by the eigenvectors with positive eigenvalues. These spaces are mutually orthogonal in $L^{2}$. From the fast growth property (ii') we see that outside a large ball of radius $R$ (we are using the $L^{2}$ norm), the term $H$ in the integrand of $F$ dominates the other term, which has quadratic growth. Thus $F<0$ outside a ball of radius $R$. (This is true only in finite dimensions; the reasoning would be false in infinite-dimensional space.) In fact, we see that $F \rightarrow-\infty$ as we go to infinity, so $F$ satisfies (PS) in $R^{N}$.

Since $H \geqslant 0$ we see that $F \leqslant 0$ on $E_{1}$. Consider the behaviour of $F$ on $E_{2}$, 
on which $Q \geqslant \lambda_{1}\left(|p|^{2}+|q|^{2}\right)$ for some $\lambda_{1}>0$. From property (i) of $H$ we see that for small $\varepsilon>0$,

$$
F(p, q) \geqslant c_{1}>0 \quad \text { on }\|(p, q)\|_{L^{2}}=\varepsilon .
$$

For convenience we will denote the pair $(p, q)$ by $z$.

From these simple properties we will now derive the existence of a nontrivial stationary point of $F$ on $R^{N}$. The point where $F$ achieves its maximum is of course such a point. But it is useless. For as we go to the limit on the approximation, the maximum of $F$ on $R^{N}$ will go to $+\infty$.

Instead we may apply the Generalized MPL of the last section. For the sphere $S^{N-k-1}$ and the map $\psi$ we take the $\varepsilon$ sphere about the origin in $E^{2}$ and the injection map. For the image of the sphere $S^{k}, \phi\left(S^{k}\right)$, we take the plane $E_{1}$. One will naturally object that this is not topologically a sphere. Well, just make it one by wrapping it around, i.e., closing it off, near infinity outside the ball of radius $R$. It is intuitively clear (and easy to prove, using degree theory) that these two spheres really link. Hence by the Generalized MPL the following number is a stationary value of $F$.

$$
c=\inf _{h} \max _{x \in B^{k+1}} F(h(x)) \geqslant c_{1}>0 .
$$

This may be much lower than the maximum of $F$, in fact we will see that it is bounded from above by a constant independent of $M$.

When closing off $E_{1}$ near infinity, let us do this in such a way that the resulting "sphere" lies in the space $E^{\prime}$, spanned by $E_{1}$ and a fixed unit eigenvector $\phi=\left(p_{0}, q_{0}\right)$ corresponding to the lowest positive eigenvalue of $Q$. We may take for $B^{k+1}$ the "ball" in $E_{1}^{\prime}$ bounded by the "sphere".

First we will obtain an upper bound for $c$ independent of $M$. If we take for $h$, in the definition of $c$, the identity (i.e., injection) map, then

$$
0<c \leqslant \max _{B^{k+1}}\left(Q-\int H\right) .
$$

This maximum is taken on at some point $z=(p(t), q(t))$ of the form

$$
z=a \phi+z_{1}, \quad z_{1} \in E_{1}
$$

and $\|z\|^{2}=a^{2}+\left\|z_{1}\right\|^{2}$. Since $H \geqslant 0$ we see, by our choice of $E_{1}$, that

$$
c \leqslant Q(z)=a^{2} \lambda_{1}+Q\left(z_{1}\right) \leqslant a^{2} \lambda_{1} \leqslant \lambda_{1}\|z\|^{2} .
$$

Furthermore we see from (14) that

$$
\int H(z)<Q(z) \leqslant \lambda_{1}\|z\|^{2} .
$$

Using (ii'), recall that $\theta \leqslant \frac{1}{2}$, we obtain easily a bounded on $\|z\|$, and hence on $c$, independent of $M$.

The last part of the argument, the limit process, is technical and we will confine the rest of our discussion to the simple case of a function $H$ which is positive homogeneous of degree 4 . In this way, at any rate, we include a complete proof of Theorem A.

Observe first that a stationary point $z=(p(t), q(t))$ in $R^{N}$ where $F$ has the 
stationary value $c$, satisfies the Euler equation

$$
\int_{0}^{2 \pi}\left[p \cdot \dot{\psi}+\phi \cdot \dot{q}-H_{p}(p, q) \cdot \phi-H_{q}() \cdot \psi\right] d t=0
$$

for every function $(\phi(t), \psi(t))$ lying in our space $R^{N}$. Here $\dot{\psi}=d \psi / d t$ etc. If we set $\phi=p, \psi=q$ we find

$$
\int 2 p \cdot \dot{q}=\int p \cdot H_{p}+q \cdot H_{q}=4 \int H
$$

by the homogeneity hypothesis. On the other hand,

$$
c=\int p \cdot \dot{q}-H
$$

and hence

$$
\int H=c \leqslant C .
$$

(The letter $C$ will be used to denote various constants independent of $M$.) By the homogeneity, it follows that

$$
\|z\|_{L^{4}} \leqslant C
$$

Next we wish to establish the estimate

$$
\|\dot{z}\|_{L^{2}} \leqslant C
$$

and to this end we set $(\phi, \psi)=(\dot{q},-\dot{p})$ (which is indeed in $\left.E_{1}\right)$ in (15). After a partial integration we find

$$
\|\dot{z}\|_{L^{2}}^{2}=\int H_{p} \cdot \dot{q}-H_{q} \cdot \dot{p} \leqslant\|\dot{z}\|_{L^{2}}\|\operatorname{grad} H\|_{L^{2}}
$$

so that

$$
\|\dot{z}\|_{L^{2}} \leqslant\|\operatorname{grad} H\|_{L^{2}} \leqslant C\|z\|_{L^{6}}^{3}
$$

Inserting (19) and (17) in the standard interpolation inequality

$$
\|z\|_{L^{\infty}} \leqslant C\left[\int|z|^{2}+|\dot{z}|^{2}\right]^{1 / 6}\|z\|_{L^{4}}^{2 / 3}
$$

we find

$$
\|z\|_{L^{\infty}} \leqslant C\left(1+\int|z|^{6}\right)^{1 / 6} \leqslant C\left(1+C\|z\|_{L^{\infty}}^{2}\right)^{1 / 6} \text { by (17). }
$$

From this it follows that $\|z\|_{L^{\infty}} \leqslant C$, and (18) then follows from (19).

From these inequalities we may draw a further conclusion. Set $\|z\|_{L^{\infty}}=\rho$. According to (16) we have for our solution $z$

$$
2 \int H=\int p \cdot \dot{q}=\int(p-\bar{p}) \cdot \dot{q}
$$

where $\bar{p}$ is the average of $p$ on $(0,2 \pi)$,

$$
\leqslant\|\dot{q}\|_{L^{2}}\|p-\bar{p}\|_{L^{2}} \leqslant C\|\dot{q}\|_{L^{2}}\|\dot{p}\|_{L^{2}} ;
$$

thus

$$
\int H \leqslant C\|\dot{z}\|_{L^{2}}^{2}
$$


On the other hand, by (19),

$$
\|\dot{z}\|_{L^{2}}^{2} \leqslant C \int|z|^{6} \leqslant C \rho^{2} \int|z|^{4} \leqslant C \rho^{2} \int H .
$$

Inserting this in (20) we find, since $\int H>0$,

$$
1 \leqslant C \rho^{2}
$$

i.e.

$$
\rho=\|z\|_{L^{\infty}} \geqslant \delta
$$

where $\delta$ is a fixed positive constant independent of $M$.

We are now in a position to carry out the limit process. Let $M \rightarrow \infty$ through the integers. By (18) we may assert that a subsequence of the $z=z_{M}$ converge weakly in $H^{1}\left(S^{1}\right)$ and also (by the Sobolev embedding theorem) uniformly to a function $z=(p(t), q(t))$ which is a solution of (13). By (21) we have $\|z\|_{L^{\infty}} \geqslant \delta>0$ and so $z$ is a nontrivial solution. A standard regularity argument then shows that the solution is smooth. Theorem B for the special case, and hence Theorem A, are proved.

Remarks. 1. By Theorem 2, for every positive integer $k$, (13) has a nontrivial solution with period $T / k$. Hence (13) has infinitely many solutions with period $T$. It is an open problem whether there are solutions of period $T$ for which $T$ is the smallest period.

2. Remarkably, Rabinowitz [54] has shown that Theorem B holds even if assumption (i) is dropped. The proof, again via a minimax argument, is based on a cohomological index theory developed by Fadell and Rabinowitz [29]. Furthermore, there exist solutions of (13) with given period $T$ and having arbitrarily large $L^{\infty}$ norms.

3. Clarke and Ekeland [20] have proved an analogue of Theorem B but under somewhat opposite conditions on $H$. They assume $H$ to be convex and to satisfy

$$
\frac{H}{|p|^{2}+|q|^{2}} \rightarrow\left(\begin{array}{c}
\infty \\
0
\end{array}\right) \text { at }\left(\begin{array}{c}
0 \\
\text { infinity }
\end{array}\right),
$$

and prove that there exists a nontrivial solution having any given $T>0$ as minimal period. Their proof is based on a different variational problem.

They introduce a different Lagrangian $F(p, q)$ for which the Euler equation is still (13). It is, essentially, a dual to the $F$ treated above. In [24] Ekeland treated a Hamiltonian behaving like that of Rabinowitz using a dual variational problem.

4. In connection with Theorem A, Ekeland and Lasry [26] consider a Hamiltonian $H$ for which the level set $\Sigma: H=1$ is a closed convex set lying in an annular region

$$
0<\frac{r}{2}<|p|^{2}+|q|^{2}<r
$$

They prove that there are at least $n$ different periodic solutions of (13) on $\Sigma$. (A closed solution curve may of course be traversed several times, but all these solutions are not considered to be different.) 
II. 5 In Remark 3 at the end of the preceding section we mentioned the use of a kind of dual Lagrangian in finding periodic solutions. In this section we will describe an adaptation of it applied to a different problem: that of finding time-periodic solutions $u(x, t)$ of a nonlinear vibrating string equation on an interval $(0, \pi)$ :

$$
u_{t t}-u_{x x}+g(u)=0, \quad 0<x<\pi ; \quad u(0, t)=u(\pi, t)=0 .
$$

Here we assume $g(0)=0$, so that $u=0$ is a solution, but we seek a nontrivial solution having a given time period $T$.

This problem bears some resemblance to the one treated in the preceding sections and similar methods are used. Until a few years ago most results for this problem were local: $g$ was assumed to be appropriately small (perhaps with a small factor $\varepsilon$ attached) and one sought solutions close to zero. In the last few years new methods have been introduced enabling one to treat large $g$. However, up to now the results are mainly confined to the case that $g$ is monotone (either increasing or decreasing). Thus we cannot yet treat the sine-Gordon equation: $g=\sin u$-for which explicit time periodic solutions are known in case the string goes to infinity in both directions.

There is also a restriction on the given time period $T$. For technical reasons we can, essentially, till now, only treat the case that $T=2 \pi / \lambda$ is a rational multiple of the length of the string, i.e., $\lambda$ is rational. The reason for this will be explained presently.

Much work has been done in the case that $g$ grows at most linearly as $|u| \rightarrow \infty$ (see [14] and [5] for some recent results). The case that $g$ grows superlinearly is, perhaps, more interesting. The main result in this case is due to Rabinowitz [52]. Assuming $g$ as above and satisfying

$$
\begin{aligned}
g(u) & =o(u) \quad \text { as } u \rightarrow 0, \\
G(u) & \leqslant \theta u g(u), \quad|u| \text { large, some } \theta \text { in }\left(0, \frac{1}{2}\right)
\end{aligned}
$$

he proved there is a nontrivial periodic solution with any given time period $T=2 \pi / \lambda, \lambda$ rational. A particular, and interesting, example is $g=u^{3}$. The solution is obtained as a nontrivial stationary point of the Lagrangian, defined for functions $u(x, t)$ satisfying the boundary condition and with time period $2 \pi / \lambda$,

$$
F(u)=\int_{0}^{2 \pi} \int_{0}^{\pi}\left[\frac{1}{2}\left(u_{x}^{2}-u_{t}^{2}\right)+G(u(x, t))\right] d x d t
$$

where

$$
G(u)=\int_{0}^{u} g(s) d s .
$$

His proof follows the scheme of the one in the preceding section and makes use of the Generalized MPL-which indeed he introduced in [52].

We will describe a different variational problem using a sort of dual Lagrangian, which leads to a simpler proof of the result. This is due to Brézis, Coron, Nirenberg [15]. The case $g=u^{3}$ admits of a particularly simple treatment. $\Omega$ will denote the period rectangle $0<x<\pi, 0<t<T$. 
First some remarks about the condition that $\lambda$ is rational. This comes from an analysis of the linear problem for $u$ with time-period $2 \pi / \lambda$ :

$$
A u=u_{t t}-u_{x x}=f, \quad u(0, t)=y(\pi, t)=0 .
$$

Fourier expansion provides a complete analysis of this-a sine series in $x$ being appropriate because of the boundary condition. Arguing formally, let us set

$$
u=\sum_{\substack{j>0 \\ k}} a_{j k} \sin j x e^{i \lambda k t}, \quad f=\sum f_{j k} \sin j x e^{i \lambda k t}
$$

Then

$$
A u=\sum\left(j^{2}-\lambda^{2} k^{2}\right) a_{j k} \sin j x e^{i \lambda k t}
$$

and, for a solution,

$$
a_{j k}=\frac{f_{j k}}{j^{2}-\lambda^{2} k^{2}}
$$

provided the denominator is not zero; if it is then we should have $f_{j k}=0$. In general, but not always, if $\lambda$ is irrational the denominators $j^{2}-\lambda^{2} k^{2}$ may be arbitrarily small for infinitely many values of $j$ and $k$. In that case if we take $u$ in $L^{2}(\Omega)$ or $H^{s}(\Omega)$ or $C^{\infty}(\bar{\Omega})$, it is very difficult to characterize the range of $A$. In general it will not be closed (in some reasonable topology) and for this reason the case of general $T$ is still intractable. We have here a small divisor problem. If $\lambda$ is rational however, for convenience let us confine ourselves to the case $\lambda=1$, the denominator, if $\neq 0$, is an integer and hence $\geqslant 1$ in absolute value. Furthermore $\left|j^{2}-k^{2}\right| \rightarrow \infty$ as $|j|+|k| \rightarrow \infty, j \neq k$. In this case, $\lambda=1$, however, the null space $N=N(A)$ is infinite dimensional, spanned by $\sin j x \cos j t, \sin j x \sin j t, j=1,2 \ldots$ (We will use $N$, sloppily, to denote the set of $C^{\infty}$ functions in $\operatorname{ker} A$, or its closure in some topology.) In fact any function in $N$ has the form

$$
q(t+x)-q(t-x)
$$

where $q$ is periodic of period $2 \pi$.

In $L^{2}(\Omega)$ the range $R(A)$ is then $N^{\perp}$ and if we factor out $N(A)$ we may consider $A^{-1}: R(A) \rightarrow R(A)$. There is a simple explicit integral formula for $A^{-1}: R(A) \rightarrow R(A)$ and it is not hard to verify the following properties of $A^{-1}$.

(i) In $L^{2}(\Omega), A^{-1}$ is compact.

(ii) $\left\|A^{-1} f\right\|_{L^{\infty}} \leqslant C\|f\|_{L^{1}}, f \in R(A)$.

(iii) Set

$$
E_{p}=\left\{v \frac{\perp}{L^{2}} N \mid v \in L^{p}\right\} \quad \text { with the } L^{p} \text { norm. }
$$

Then $A^{-1}: E_{p} \rightarrow L^{p^{\prime}}$ is compact for $1<p<\infty$. Here $1 / p+1 / p^{\prime}=1$.

The result in [15] is slightly more general than that in [52]. 
TheOREM [15]. Let $g$ be as above and monotone increasing and let $\lambda$ be rational. Assume

$$
\lim _{|t| \rightarrow \infty} \frac{g(t)}{t}=\infty, \quad \frac{1}{2} g(t)-G(t) \geqslant \alpha|g(t)|-C
$$

$\forall t$ and some constants $C, \alpha>0$. Then there is a nontrivial solution $u \in L^{\infty}$ of (22) with time period $2 \pi$.

For $g$ strictly increasing and $C^{\infty}$, it was shown in [14] and [52] that $u \in C^{\infty}(\bar{\Omega})$.

The variational approach of [15] was motivated by the work in [20], [24]. We will assume $g$ is strictly increasing; denote $g^{-1}$ by $h$ and set

$$
H(v)=\int_{0}^{v} h(s) d s .
$$

First we rewrite equation (22)-arguing formally. Suppose $u$ is a solution; decompose it

$$
u=u_{1}+u_{2}
$$

$u_{1} \in R(A), u_{2} \in N$, and set

$$
v=g\left(u_{1}+u_{2}\right)
$$

The equation then has the form

$$
A u_{1}+v=0
$$

So $v \in R(A)$,

$$
u_{1}+u_{2}=h(v)
$$

and the equation is equivalent to

$$
u_{1}+A^{-1} v=0
$$

or, after adding $u_{2}$ to both sides

$$
A^{-1} v+h(v)=u_{2} \in N .
$$

To this form we now associate the following Lagrangian defined on $R(A)$ alone, i.e. we throw away the infinite-dimensional nullspace $N$,

$$
F(v)=\iint_{\Omega}\left[\frac{1}{2} v \cdot A^{-1} v+H(v)\right], \quad v \in R(A) .
$$

Note, indeed, that if $v \in R(A)$ is a stationary point of $F$ then

$$
0=F^{\prime}(v)(\zeta)=\iint_{\Omega}\left(A^{-1} v+h(v)\right) \zeta, \quad \forall \zeta \in R(A) .
$$

It follows that $A^{-1} v+h(v)=u_{2}$ belongs to $R^{\perp}=N$. Reversing the steps above we obtain a solution of (22). Thus the $u_{2}$ component of $u$ appears as a kind of Lagrange multiplier.

The Lagrangian (24) looks much more tractable than (23) since $A^{-1}$ is compact. The case $g(u)=|u|^{q} u, q \neq 1$, is particularly simple. Consider $g=u^{3}$ (this proof works as well for other values of $q$ ). In this case $H=$ const $|v|^{4 / 3}$. 
We may obtain a solution via a still different variational problem. Namely

$$
\min _{\substack{v \in E_{4 / 3} \\\|v\|_{L^{4 / 3}<1}}} \iint_{\Omega} v \cdot A v .
$$

Because of the compactness property (iii), with $p=4 / 3$, and the fact that $v \cdot A^{-1} v$ may be negative one finds that the minimum occurs at some point $v$, which is necessarily on the boundary of the ball. Consequently for some Lagrange multiplier $\lambda>0$ we have

$$
A^{-1} v+\lambda|v|^{-2 / 3} v \in N
$$

We want $\lambda=1$, and this is achieved simply by stretching $v$. Unfortunately this argument works only in the case that $g$ is homogeneous.

As an exercise for the reader we suggest reproving the result for $g=u^{3}$ using the mountain pass lemma in the space $E_{4 / 3}$.

The general case is treated in [15] with the aid of the mountain pass lemma in the space $E_{1}$. In order to work in this space the function $h(v)$ is first changed for $|v|$ large so as to be constant on the intervals $v>k ; v \leqslant-k$. The space $L^{1}$ is not reflexive, making it difficult to verify (PS). In fact we were not able to, and we were led to a modified form of the condition. The modification is employed in just the same way as condition (PS) and we believe it will prove useful in other problems. In this form, $c$ is a fixed real number, and we consider a real function $F$ defined on a Banach space $X$ which is Gâteaux differentiable (in our particular case in [15] the function is not Fréchet differentiable).

CONDITION $(\mathrm{PS})_{\mathrm{c}}$. If there is a sequence $\left\{u_{j}\right\}$ in $X$ such that

$$
F\left(u_{j}\right) \rightarrow c \text { and } F^{\prime}\left(u_{j}\right) \rightarrow 0 \text { strongly, }
$$

then $c$ is a critical value of $F$.

Clearly (PS) $\Rightarrow(\mathrm{PS})_{\mathrm{c}}$ for every $c$.

The mountain pass lemma then holds in the following form, [15],

LEMMA. Let $F$ be Gâteaux differentiable and $F^{\prime}: X \rightarrow X^{*}$ continuous from the strong topology of $E$ into the weak ${ }^{*}$ topology of $E^{*}$. Assume that there is an open neighbourhood $U$ of 0 , a point $u_{0} \notin \bar{U}$, and a constant $\rho$ such that $F\left(u_{0}\right), F(0)<\rho \leqslant F(u)$ for all $u \in \partial U$. Assume $F$ satisfies (PS) for

$$
c=\inf _{P} \max _{u \in P} F(u) \geqslant \rho .
$$

Here $P$ is a continuous path from 0 to $u_{0}$, and the inf is taken over all such paths. Then $c$ is a critical value of $F$.

\section{Local theory}

III.1 This part will be concerned with the local study of equation (1). We will suppose as before that the mapping $F$ is from one Banach space $X$ to another, $Y$, and that

$$
F\left(u_{0}\right)=y_{0}
$$


One wishes to study the set of solutions near $u_{0}$ of a perturbed equation

$$
F(u)=y \quad \text { near } y_{0}
$$

-or $F$ may depend on one or more parameters $\lambda$

$$
F(u, \lambda)=y, \quad F\left(u_{0}, \lambda_{0}\right)=y_{0} .
$$

Our remarks will refer to the case that the implicit function theorem (IFT) is not applicable.

In case $F$ is a Fredholm operator the well-known Lyapounoff-Schmidt procedure (see for example Chapter 3 in [47]) reduces the local study to one in finite-dimensional spaces. In case dim ker $F^{\prime}\left(u_{0}\right)=d$, codim Range $F^{\prime}\left(u_{0}\right)$ $=d^{\prime}$, the procedure leads to a mapping from $R^{d}$ to $R^{d^{\prime}}$. But the problem remains a difficult one; many different kinds of phenomena can occur.

One procedure in the finite-dimensional case, is to try to find a suitable local change of variables after which the (finite-dimensional) map $F$ reduces to some simple canonical form admitting rather easy analysis. For example, if $F$ is a real smooth function defined on $R^{d}$ near the origin $F(0)=0$, the simplest case is the case: $d F(0) \neq 0$. Then by the IFT we can find new coordinates so that $F$ has the form $F(x)=x_{1}$. If $d F(0)=0$, the next simple generic case, is to suppose that 0 is a nondegenerate critical point of $F$. In that case the well-known Morse lemma says that after a suitable change of coordinates, $F$ is equal to a homogeneous quadratic function. More generally, the theory of singularities of differentiable maps, stability of maps and, in particular, catastrophe theory of Thom and Mather is very useful in the study of qualitative behaviour of solutions of (26). In this theory one makes changes of variables $(x, \lambda)$ and $y$ to reduce the mappings to simple forms. Sometimes one wishes to distinguish the parameter variable $\lambda$, and not permit general change of variables which mix those with $x$. A suitable corresponding form of the theory has been developed by Golubitsky and Schaeffer [33], and applied to problems in elasticity as well as others.

Local problems in which $F$ is a Fredholm operator, but Range $F^{\prime}\left(u_{0}\right) \neq Y$, are often called bifurcation problems. Even in such problems, global topological techniques are very useful. We describe one example:

A classical bifurcation problem is one concerning periodic solutions for ordinary differential equations; here $x$ is a vector in $R^{n}$.

$$
\frac{d x}{d t}=F(x, \lambda)
$$

where $F$ depends smoothly on $x$ and a real parameter $\lambda$, near $\lambda=0$, and

$$
F(0, \lambda) \equiv 0, \quad \forall \lambda \text {. }
$$

We are interested in finding nontrivial periodic solutions near the origin for $|\lambda|$ small, which bifurcate from the origin. Let

$$
F_{x}(0, \lambda)=L(\lambda)
$$

and assume the matrix $L(0)$ is nonsingular. Then for $|\lambda|$ small, the only stationary point of (27) is $x=0$. It is easy to see that no periodic solutions will bifurcate from the origin unless $L(0)$ has a purely imaginary eigenvalue. 
Suppose $i \beta, \beta>0$, is an eigenvalue of $L(0)$. Then the linearized equation

$$
\frac{d x}{d t}=L(0) x
$$

has a nonconstant periodic solution of period $2 \pi \beta^{-1}$. (For $|\lambda|$ small, $L(\lambda)$ has a unique eigenvalue $\alpha(\lambda)+i \beta(\lambda)$ which is close to $i \beta$, and it depends continuously on $\lambda$.) We are interested in finding nonconstant periodic solutions with period close to $2 \pi \beta^{-1}$ in case $|\lambda|$ is small.

In this direction there is the classical result of Hopf. Here is a well-known formulation (see [41] for a general survey).

Hopf Bifurcation TheOREM. Let $i \beta$ be as above and assume that no other integral multiple of $i \beta$ is an eigenvalue of $L(0)$. Assume that $\alpha(\lambda) \neq 0$ for $\lambda \neq 0$ and changes sign as $\lambda$ crosses 0 . Then for $|\lambda|$ small, there exists a nontrivial small periodic solution of (27) with period close to $2 \pi \beta^{-1}$.

Alexander and Yorke [2] discovered a generalization. Assume as before that $i \beta, \quad \beta>0$, is an eigenvalue of $L(0)$ and that $\operatorname{Mult}(i \beta)=$ $\left\{i k_{1} \beta, i k_{2} \beta, \ldots, i k_{r} \beta\right\}, 1 \leqslant k_{1} \leqslant \cdots \leqslant k_{r}$, positive integers, are all the eigenvalues of $L(0)$ which are positive integral multiples of $i \beta_{r}$ counted with multiplicity. For $|\lambda|$ small there is then a unique set of $r$ eigenvalues $\alpha_{j}(\lambda)+$ $i \beta_{j}(\lambda), j=1, \ldots, r$ close to the set, and varying continuously with $\lambda$.

Extension of THe Hopf Bifurcation Theorem [2]. Assume that for $\lambda \neq 0$, $\alpha_{j}(\lambda) \neq 0, j=1, \ldots, r$. Let $r^{+}\left(r^{-}\right)$be the number of these which are positive for $\lambda>0(\lambda<0)$. Assume that $r=r^{+}-r^{-}$, the number of changes of signs of the $\alpha_{j}$ as $\lambda$ passes through 0 , is odd. Then for $|\lambda|$ small there is a nontrivial, small periodic solution of (27) with period close to $2 \pi \beta^{-1}$.

The proof in [2] relies on some topological machinery. Subsequently Ize [35] presented a simpler proof which uses less topology. It makes use of the Hopf map of $S^{3}$ to $S^{2}$. [2] and [35] also contain more global results, concerning a connected family of periodic solutions.

The paper [35] has many results on bifurcation in particular when a real or complex parameter is involved. In papers [1], [3] further topological tools are used in analyzing bifurcation. Applications of bifurcation theory may be found in [55].

III.2 As we mentioned in $\$ I .1$ the implicit function theorem may fail because of the "loss of derivatives" on applying $F\left(u_{0}\right)^{-1}$-a situation which is common in nonelliptic problems. For example, consider the wave equation for a function $u(x, t), x \in R^{n}, t \in R^{1}$,

$$
u_{t t}-\Delta_{x} u=f, \quad u=u_{t}=0 \quad \text { at } t=0 .
$$

If, say, $f$ has compact support then for $f$ in the Sobolev space $H^{1}$ the solution $u$ belongs to $H^{s+1}$-not to $H^{s+2}$. If we consider then a perturbation problem of the form

$$
F(u)=u_{t t}-f\left(u_{x_{k}}, u_{t}, u_{x_{i} x_{j}}\right)=y ; \quad u=u_{0}=0 \quad \text { at } t=0
$$


which is hyperbolic, and $u=0$ is a solution for $y=0$, and wish to solve this for $y$ small, the implicit function theorem is not applicable.

In [44], Nash introduced a procedure for attacking local problems in which such a loss of derivatives occurs. Since then several variations have been developed. In particular Moser [42] introduced a scheme which is based on the Newton iteration scheme for solving a nonlinear problem. This method is now commonly called the Nash-Moser technique. See also Hörmander [34] for another modification of the Nash method. This technique is to my mind a truly fundamental development in the study of nonlinear problems. For the first time it enabled one to solve problems involving "loss of derivatives", which up to then had appeared completely intractable.

Before describing the Nash-Moser procedure let us first recall the Newton iteration scheme for solving a problem $F(u)=g$ where $F$ maps $R^{N} \rightarrow R^{N}$. Suppose $F\left(u_{0}\right)=y_{0}$ and $y$ is close to $y_{0}$. Assuming $F^{\prime}\left(u_{0}\right)$ is invertible, the Picard iteration scheme is the following,

$$
u_{p+1}=u_{p}+F^{\prime}\left(u_{0}\right)^{-1}\left(y-F\left(u_{p}\right)\right), \quad p=0,1, \ldots,
$$

and it converges like a geometric series. Newton's scheme is

$$
u_{p+1}=u_{p}+F^{\prime}\left(u_{p}\right)^{-1}\left(y-F\left(u_{p}\right)\right), \quad p=0,1, \ldots,
$$

assuming that $F^{\prime}(u)^{-1}$ exists for $u$ near $u_{0}$. This scheme converges much more rapidly than Picard's.

In case the operators $F^{\prime}(u)^{-1}$ "lose derivatives", $u_{p+1}$ is less regular than $u_{p}$, and so there is hope for convergence in a function space with some prescribed regularity.

The Nash-Moser scheme makes use of smoothing operators depending on a parameter. To be specific, say we are working in Sobolev spaces $H^{s}$ of some manifold and denote the corresponding norms by \|\|$_{s}$. For $\theta>1, S_{\theta}$ is supposed to be a linear operator mapping $H^{s}$ into $C^{\infty}, \forall s$, and satisfying

$$
\begin{aligned}
& \left\|S_{\theta} u\right\|_{k} \leqslant C \theta^{k-j}\|u\|_{j} \text { for } j<k, \\
& \left\|\left(I-S_{\theta}\right) u\right\|_{k} \leqslant C \theta^{k-j}\|u\|_{j} \text { for } j>k \text {. }
\end{aligned}
$$

The scheme is to choose a sequence $\theta_{j}=2^{\alpha^{j}}, 1<\alpha<2$, and work with a modified Newton scheme

$$
u_{p+1}=u_{p}+S_{\theta_{p}} F^{\prime}\left(u_{p}\right)^{-1}\left(y-F\left(u_{p}\right)\right) .
$$

Because of the smoothing, all the iterates are $C^{\infty}$-functions. Under suitable conditions on $F$-in particular one needs $F^{\prime}(u)$ to be invertible not only at $u_{0}$ but nearby-one shows that the scheme converges. The error introduced by the smoothing operators is compensated for by the rapid convergence of the Newton scheme. We refer the reader to [43], Chapter 2 in [57] and the last chapter in [47].

III.3 Recently Klainerman [36] has adapted this scheme to study the following problem posed by F. John. Consider the initial value problem (we 
will limit ourselves to a special case) for $u(x, t)$

$$
\begin{aligned}
& u_{t t}-a^{i j}(\nabla u) u_{x_{i} x_{j}}=0, \\
& u=f_{0}, \quad u_{t}=g_{0} \quad \text { at } t=0 .
\end{aligned}
$$

The coefficients $a^{i j}$ are assumed to be smooth and to satisfy

$$
a^{i j}-\delta^{i j}=b^{i j} \text { and } b^{i j}(0)=0 .
$$

The functions $f_{0}, g_{0} \in C^{\infty}$ and (with many derivatives) are assumed to be small. For convenience let us suppose they have compact support. The problem is whether there exist global (i.e. for all time) smooth solutions. If we do not assume $f_{0}, g_{0}$ to be small then simple examples show that the answer can be no. On the other hand, in one space dimension, even if $f_{0}, g_{0}$ are small but not zero, if the $a^{i j}$ are not constant, one knows that shocks will occur. Namely, the solution will exist and be smooth for small $t$, but eventually singularities must develop. In higher dimensions there is more space for the characteristics (which carry singularities) to spread and so one might hope that smooth solutions may exist for all time.

Klainerman proved this to be the case if $n \leqslant 6$.

Naturally one is interested in knowing what happens for smaller $n$, in particular $n=3$. Very recently F. John has shown that for the equation

$$
u_{t t}-\frac{\Delta u}{1-u_{t}}=0
$$

no matter how small are $\left(f_{0}, g_{0}\right) \not \neq 0$, there is no global smooth solution for all time.

We will conclude these lectures by a brief and very informal description of Klainerman's procedure. The method we describe is the first one he devised. In the published paper [36] he used a modification. The proof is highly technical so we can only sketch some of the ideas.

A classical result is that one can solve the initial value problem, and obtain a $C^{\infty}$ solution in a small time interval, $0 \leqslant t \leqslant T$, where $T$ depends only on the following (all integral norms are over the space variable in $R^{n}$ ):

$$
\sum_{|\alpha|<[n / 2]+2}\left\|\partial^{\alpha} f_{0}\right\|_{L^{2}}+\sum_{|\alpha|<[n / 2]+1}\left\|\partial^{\alpha} \sigma_{0}\right\|_{L^{2}}
$$

Here $\alpha$ is a multi-index of nonnegative integers $\alpha=\left(\alpha_{1}, \ldots, \alpha_{n}\right), \partial^{\alpha}=$ $\partial_{1}^{\alpha_{1}}, \ldots \partial_{n}^{\alpha_{n}}, \partial_{j}=\partial / \partial x_{j}$, these involve only space derivatives. Thus to find a global smooth solution it suffices to find a solution for which the following norms (in $R^{n}$ ) are bounded in $0 \leqslant t \leqslant T$ :

$$
\sum_{|\alpha| \leqslant[n / 2]+2}\left\|\partial^{\alpha} u\right\|_{L^{2}}+\sum_{|\alpha| \leqslant[n / 2]+1}\left\|\partial^{\alpha} u_{t}\right\|_{L^{2}} \leqslant C(T)
$$

for every $T ; C(T)$ may however grow as $T \rightarrow \infty$ in any way.

(a) In order to attack the problem we expect to make use of results for corresponding linear equations. Consider a linear problem

$$
u_{t t}-a^{i j}(x, t) u_{x_{i} x_{j}}+b^{i} u_{x_{i}}=g
$$


with the matrix $a=\left\{a^{i j}\right\}$ positive definite. Bounds on the $L^{2}$ norms (in $R^{n}$ ) of derivatives of the solution are usually obtained with the aid of energy estimates. If one defines the energy at time $t$ as

$$
\int_{R^{n}} u_{t}^{2}+a^{i j} u_{x_{i}} u_{x_{j}}
$$

then one finds, using Green's theorem, that

$$
\begin{aligned}
\frac{d}{d t} E(t) & =\int a_{t}^{i j} u_{i} u_{j}-a_{x_{j}}^{i j} u_{i} u_{t}+2 g u_{t}-2 b^{i} u_{i} u_{t} \\
& \leqslant\left(\max _{R^{n}}|\nabla a|+\max _{R^{n}}|b|\right) E+2\|g\|_{L^{2}} E^{1 / 2}
\end{aligned}
$$

-in rather obvious notation. Integrating from 0 to time $t$ we find

$$
E^{1 / 2}(t) \leqslant c+\int_{0}^{t} \exp \left[\int_{0}^{s}\left(\max _{x}|\nabla a(x, \sigma)|+\max _{x}|b|\right) d \sigma\right]\|g\|_{L^{2}}(s) d s .
$$

If we want the energy to be finite for all time it seems reasonable to require that for all $t$,

$$
\max _{x}(|\nabla a|+\max |b|)(t) \leqslant \frac{K}{(1+t)^{1+\varepsilon}} \quad \text { for some } \varepsilon>0 .
$$

This linear problem is the kind that will arise in the Nash-Moser scheme when we linearize about some iterate $v$. In view of the way the coefficients in the linear operator depend on $v$ it seems reasonable to work with functions whose derivatives decay as $t \rightarrow \infty$, uniformly in $x$.

(b) The next crucial fact is that for solutions of the classical wave equation such decay estimates exist. Namely, suppose $u$ satisfies

$$
u_{t t}-\Delta u=0, \quad u=0, \quad u_{t}=g \quad \text { at } t=0 .
$$

Then

$$
\begin{aligned}
& |u(x, t)| \leqslant C t^{(1-n) / 2} \sum_{|\alpha|<(n-1) / 2}\left|\partial^{\alpha} g\right|_{L^{1}}, \\
& |\nabla u(x, t)| \leqslant C t^{(1-n) / 2} \sum_{|\alpha|<(n+1) / 2}\left|\partial^{\alpha} g\right|_{L^{1}} .
\end{aligned}
$$

In addition, by the standard energy estimates (applied to spatial derivatives of $u$ ), and by the Sobolev embedding theorem, we have

$$
|\nabla u(x, t)| \leqslant C \sum_{|\alpha|<n / 2+1}\left\|\partial^{\alpha} g\right\|_{L^{2}}
$$

This leads to a corresponding decay for a solution of the inhomogeneous equation

$$
u_{t t}-\Delta u=g, \quad u=u_{t}=0 \quad \text { at } t=0,
$$

in case $g$ decays in time. In terms of the appropriate solution operator $V(t), u$ is given by (here we suppress the space variables)

$$
u(t)=\int_{0}^{t} V(t-s) g(s) d s
$$


so that

$$
|\nabla u(x, t)| \leqslant\left|\int_{0}^{t / 2} \nabla V(t-s) g(s) d s\right|+\left|\int_{1 / 2}^{t} \nabla V(t-s) g(s) d s\right| .
$$

We have divided the $t$ integral into two parts. In the first we use the estimate (29) which is useful for large, not small, $t$, while in the second we use (30). We thus obtain the estimate (where we have thrown something away)

$$
\begin{aligned}
|\nabla u(x, t)| \leqslant & C t^{(1-n) / 2} \int_{0}^{t / 2} \sum_{|\alpha|<n / 2+1}\left|\partial^{\alpha} g\right|_{L^{1}}(s) d s \\
& +C \int_{t / 2}^{t} \sum_{|\alpha|<n / 2+1}\left\|\partial^{\alpha} g\right\|_{L^{2}} d s .
\end{aligned}
$$

Since we want solutions to decay, this suggests introducing decay norms, for $k \geqslant 1$,

$$
\begin{aligned}
|u|_{k, r, L^{1}} & =\sup _{t>0}(1+t)^{k} \sum_{|\alpha|<r}\left|\partial^{\alpha} u\right|_{L^{1}}, \\
\|u\|_{k, r} & =\sup (1+t)^{k} \sum_{|\alpha|<r}\left\|\partial^{\alpha} u\right\|_{L^{2 .}}
\end{aligned}
$$

Then from the preceding inequality we find for $k>1$,

$$
|\nabla u(x, t)| \leqslant C t^{(3-n) / 2}|g|_{0, n / 2+1, L^{1}}+C(1+t)^{1-k}\|g\|_{k, n / 2+1} .
$$

Now in our application of this to be the nonlinear problem, via the Nash-Moser scheme, the function $g$ will involve products of derivatives of iterates. In view of energy estimates, we cannot expect the $L^{2}$ norms of these derivatives to decay. They may only, at best, remain bounded. So if the max norm in $R^{n}$ of the derivatives decay like $O\left(t^{-k}\right)$ then, at best, $\|g\|_{k, n / 2+1}$ will be bounded. But then the last term on the right of (31) decays only like $O\left(t^{1-k}\right)$. Thus there is a loss in decay-as well as the usual loss in smoothness. Furthermore, the first term on the right of (31) shows that one cannot expect to establish faster decay than $O\left(t^{(3-n) / 2}\right)$. In particular, for $n=3$, this doesn't give any decay at all.

(c) Now we turn to the iteration scheme. To overcome the loss in decay (as well as regularity), Klainerman introduces a modified "smoothing operator" in the Nash-Moser scheme: His operator involves smoothing and, at the same time, cut off after large time: If $\zeta(t) \geqslant 0$ is a $C^{\infty}$ function on $t \geqslant 0, \zeta \equiv 1$ in $0 \leqslant t \leqslant 1, \zeta(t)=0$ for $t>2$, define the operator

$$
C_{\theta}=\text { multiplication by } \zeta\left(\frac{t}{\theta}\right), \quad \theta>1 \text {. }
$$

The new "smoothing operator" will be a product

$$
C_{\theta} \cdot S_{\theta^{\prime}}, \quad \theta, \theta^{\prime}>1
$$

Next, the iteration scheme used is a modification of the Nash-Moser scheme. For a suitable choice of $\theta_{p} \rightarrow \infty$ and $\theta_{p}^{\prime} \rightarrow \infty$, set

$$
\bar{S}_{p}=C_{\theta_{p}} S_{\theta_{p}^{\prime}}
$$


In fact $\theta_{p+1}=\theta_{p}^{\tau}$ for some $\tau$ in $1<\tau<2$, and $\theta_{p}^{\prime}=\theta_{p}^{e}$ for some small $\varepsilon>0$. The $(p+1)$ st iterate is defined as the solution of

$$
L_{p}\left(u_{p+1}-u_{p}\right)=y-\bar{S}_{p} F\left(u_{p}\right)
$$

with zero Cauchy data on $t=0$. Here $L_{p}$ is the linear operator (a modification of $F^{\prime}\left(u_{p}\right)$ )

$$
L_{p}=\partial_{t t}-\Delta-\bar{S}_{p}\left[b^{i j}\left(\nabla u_{p}\right)\right] \partial_{i} \partial_{j}+\bar{S}_{p}\left(u_{p_{x_{i} x_{j}}} b^{i j}\left(\nabla u_{p}\right)\right) \cdot \nabla
$$

is rather obvious notation.

Making use of the energy estimates and the decay estimates described above (applied to spatial derivatives of the iterates) one establishes after much work a series of recursive estimates. This involves the use of still more norms and at this point we have to refer to [36] for the details.

Our description of Klainerman's treatment is perhaps too sketchy to be useful. It has been included as an indication of the flexibility of the NashMoser method. In particular, I want to call attention to the use of the "smoothing operators"-they need not merely smooth, they might operate in some other way-in this case by cut off for large time.

\section{REFERENCES}

1. J. C. Alexander, Bifurcation of zeros of parametrized functions (to appear).

2. J. C. Alexander and J. A. Yorke, Global bifurcation of periodic orbits, Amer. J. Math. 100 (1978), 263-292.

3. Calculating bifurcation invariants as elements in the homotopy of the general linear group, J. Pure Appl. Algebra 13 (1978), 1-8.

4. H. Amann and E. Zehnder, Nontrivial solutions for a class of nonresonance problems and applications to nonlinear differential equations (to appear).

5. , Multiple periodic solutions for a class of nonlinear autonomous wave equations (to appear).

6. A. Ambrosetti and P. H. Rabinowitz, Dual variational methods in critical point theory and applications, J. Funct. Anal. 14 (1973), 349-381.

7. A. Ambrosetti and G. Mancini, Sharp nonuniqueness results for some nonlinear problems, Nonlinear Anal. Theory Math. Appl. 3 (1979), 635-645.

8. V. Benci, Some critical point theorems and applications, Comm. Pure Appl. Math. 33 (1980), 147-172.

9. , On the critical point theory for indefinite functionals in the presence of symmetries, Ist. Mat. Appl. U. Dini, Univ. di Pisa, March 1980.

10. V. Benci and P. H. Rabinowitz, Critical point theorems for indefinite functionals, Invent. Math. 52 (1979), 241-273.

11. M. S. Berger, Nonlinearity and functional analysis, Academic Press, New York, 1978.

12. Yu. G. Borisovich, V. G. Zvyagin and Yu. I. Sapronov, Nonlinear Fredholm maps and the Leray-Schauder theorem, Uspehi Mat. Nauk 32 (1977), 3-54; English transl. in Russian Math. Surveys 32 (1977), 1-54.

13. H. Brézis and R. E. L. Turner, On a class of superlinear elliptic problems, Comm. Partial Differential Equations 2 (1977), 601-614.

14. H. Brézis and L. Nirenberg, Forced vibrations for a nonlinear wave equation, Comm. Pure Appl. Math. 31 (1978), 1-30.

15. H. Brézis, J. M. Coron and L. Nirenberg, Free vibrations for a nonlinear wave equation and a theorem of P. Rabinowitz, Comm. Pure Appl. Math. 33 (1980), 667-684. 
16. A. Castro, $A$ two point boundary value problem with jumping nonlinearities, Proc. Amer. Math. Soc. (to appear).

17. A. Castro and A. C. Lazer, Critical point theory and the number of solutions of a nonlinear Dirichlet problem, Ann. Mat. Pura Appl. (IV) 70 (1979), 113-137.

18. K. C. Chang, The obstacle problem and partial differential equations with discontinuous nonlinearities, Comm. Pure Appl. Math. 33 (1980), 117-146.

19. S_ Solutions of asymptotically linear operator equations via Morse theory, Comm. Pure Appl. Math. (to appear).

20. F. H. Clarke and I. Ekeland, Hamiltonian trajectories having prescribed minimal period, Comm. Pure Appl. Math. 33 (1980), 103-116.

21. C. C. Conley, Isolated invariant sets and the Morse index, CBMS Regional Conf. Ser. in Math., no. 38, Amer. Math. Soc., Providence, R. I., 1978.

22. D. DeFigueiredo, P. L. Lions and R. D. Nussbaum, Estimations à priori pour les solutions positives de problèmes elliptiques semilinéaires, C. R. Acad. Sci. Paris Ser. A. 290 (1980), 217-220.

23. I. Ekeland, On the variational principle, J. Math. Anal. Appl. 47 (1974), 324-353.

24. Periodic solutions of Hamiltonian equations and a theorem of P. Rabinowitz, J. Differential Equations 34 (1979), 523-534.

25. I. Ekeland and R. Témam, Convex analysis and variational problems, Studies in Math. and Appl., Vol. 1, North-Holland, Amsterdam, American Elsevier, New York, 1976.

26. I. Ekeland and J. M. Lasry, On the number of periodic trajectories for a Hamiltonian flow on a convex energy surface, MRC Technical Report 2050, Math. Research Center, Univ. of Wisconsin, 1980.

27. K. D. Elworthy and A. J. Tromba, Differential structures and Fredholm maps, Proc. Sympos. Pure Math. (Berkeley, California, 1968), vol. 15, Amer. Math. Soc., Providence, R. I., 1970, pp. 45-94.

28. Degree theory on Banach manifolds, Proc. Sympos. Pure Math., vol. 18, part 1, Nonlinear functional analysis, Amer. Math. Soc., Providence, R. I., 1970, pp. 86-94.

29. F. R. Fadell and P. H. Rabinowitz, Generalized cohomological index theories for Lie group actions with an application to bifurcation questions for Hamiltonian systems, Invent. Math. 45 (1978), 134-174.

30. F. B. Fuller, An index of fixed point type for periodic orbits, Amer. J. Math. 89 (1967), 133-148.

31. K. Geba and A. Granas, Infinite dimensional cohomology theories, J. Math. Pure Appl. 52 (1973), 145-270.

32. B. Gidas and J. Spruck, A priori bounds for positive solutions of nonlinear elliptic equations, Comm. Partial Differential Equations (submitted).

33. M. Golubitsky and D. Schaeffer, $A$ theory for imperfect bifurcation via singularity theory, Comm. Pure Appl. Math. 32 (1979), 21-98.

34. L. Hörmander, The boundary value problems of physical geodesy, Arch. Rational Mech. Anal. 62 (1976), 1-52.

35. J. Ize, Bifurcation theory for Fredholm operators, Mem. Amer. Math. Soc. No. 174 (1976).

36. S. Klainerman, Global existence for nonlinear wave equations, Comm. Pure Appl. Math. 33 (1980), 43-101.

37. M. A. Krasnoselskii, Topological methods in the theory of nonlinear integral equations, Macmillan, New York, 1964.

38. J. Leray and J. Schauder, Topologie et équations fonctionelles, Ann. Sci. École Norm. Sup. 51 (1934), 45-78.

39. N. G. Lloyd, Degree theory, Cambridge Tracts in Math., No. 73, Cambridge Univ. Press, London, 1978.

40. J. Mallet-Paret and J. A. Yorke, Snakes: Oriented families of periodic orbits, their sources, sinks and continuation (to appear).

41. J. E. Marsden and M. McCracken, The Hopf bifurcation and its applications, Appl. Math. Sciences, Vol. 19, Springer-Verlag, New York, 1976.

42. J. Moser, A new technique for the construction of solutions of nonlinear differential equations, Proc. Nat. Acad. Sci. U.S.A. 47 (1961), 1824-1831.

43. , A rapidly convergent iteration method and nonlinear partial differential equations. I, 
II, Ann. Scuola Norm. Sup. Pisa 20 (1966), 265-315; 499-535.

44. J. Nash, The imbedding problem for Riemannian manifolds, Ann. of Math. (2) 63 (1956), 20-63.

45. W. M. Ni, Some minimax principles and their applications in nonlinear elliptic equations, J. d'Analyse Math. 37 (1980), 248-275.

46. L. Nirenberg, An application of generalized degree to a class of nonlinear problems, 3rd Colloq. Anal. Fonct. Liège Centre Belge de Rech. Math., 1971, pp. 57-73.

47. __ Topics in nonlinear functional analysis, Lecture Notes, Courant Inst., 1974.

48. __ Remarks on nonlinear problems, The Chern Symposium, 1979 (W. Y. Hsiang et al., eds.) Springer-Verlag, Berlin and New York, 1980, pp. 189-197.

49. R. S. Palais, Critical point theory and the minimax principle, Proc. Sympos. Pure Math., vol. 15, Amer. Math. Soc., Providence, R. I., 1970, pp. 185-212.

50. P. H. Rabinowitz, Variational methods for nonlinear eigenvalue problems (CIME, Verona, 1974), Ediz. Cremonese Rome, 1974, pp. 141-195.

51. Théorie du degré topologique et applications à des problèmes aux limites nonlinéaires, Lecture Notes, Analyse Numerique Fonctionelle, Univ. Paris VI, 1975. Free vibrations for a semilinear wave equation, Comm. Pure Appl. Math. 31 (1978), 31-68.

53. , Periodic solutions of Hamiltonian systems, Comm. Pure Appl. Math. 31 (1978), 157-184.

54. A variational method for finding periodic solutions of differential equations. Nonlinear Evolution Equations (M. G. Crandall, ed.),Academic Press, New York, 1978, pp. 225-251.

55. D. H. Sattinger, Topics in stability and bifurcation theory, Lecture Notes in Math. no. 309, Springer-Verlag, Berlin and New York, 1973.

56. M. Schechter, Principles of functional analysis, Academic Press, New York, 1971.

57. J. Schwartz, Nonlinear functional analysis, Gordon and Breach, New York, 1969.

58. J. Sylvester, Ph.D. Thesis, Courant Inst. Math. Sci., New York Univ., 1980.

Courant Institute of Mathematical Sciences, New York University, New York, New YORK 10012 